\title{
ANALYSIS OF A TWO-SCALE, LOCALLY CONSERVATIVE SUBGRID UPSCALING FOR ELLIPTIC PROBLEMS*
}

\author{
TODD ARBOGAST ${ }^{\dagger}$
}

\begin{abstract}
We present a two-scale theoretical framework for approximating the solution of a second order elliptic problem. The elliptic coefficient is assumed to vary on a scale that can be resolved on a fine numerical grid, but limits on computational power require that computations be performed on a coarse grid. We consider the elliptic problem in mixed variational form over $W \times \mathbf{V} \subset L^{2} \times H$ (div). We base our scale expansion on local mass conservation over the coarse grid. It is used to define a direct sum decomposition of $W \times \mathbf{V}$ into coarse and "subgrid" subspaces $W_{c} \times \mathbf{V}_{c}$ and $\delta W \times \delta \mathbf{V}$ such that (1) $\nabla \cdot \mathbf{V}_{c}=W_{c}$ and $\nabla \cdot \delta \mathbf{V}=\delta W$, and (2) the space $\delta \mathbf{V}$ is locally supported over the coarse mesh. We then explicitly decompose the variational problem into coarse and subgrid scale problems. The subgrid problem gives a well-defined operator taking $W_{c} \times \mathbf{V}_{c}$ to $\delta W \times \delta \mathbf{V}$, which is localized in space, and it is used to upscale, that is, to remove the subgrid from the coarse-scale problem. Using standard mixed finite element spaces, two-scale mixed spaces are defined. A mixed approximation is defined, which can be viewed as a type of variational multiscale method or a residual-free bubble technique. A numerical Green's function approach is used to make the approximation to the subgrid operator efficient to compute. A mixed method $\pi$-operator is defined for the two-scale approximation spaces and used to show optimal order error estimates.
\end{abstract}

Key words. second order elliptic, two-scale expansion, upscaling, subgrid, mixed method, variational multiscale method, numerical Green's function

AMS subject classifications. 65N15, 65N30, 35J20

DOI. $10.1137 /$ S0036142902406636

1. Introduction. Many mathematical models and numerical schemes have appeared in the literature that can capture fine-scale phenomena on coarse scales or grids. This is the essence of upscaling. The change-of-scale problem goes back to the beginning of mathematical modeling; however, research on it has recently seen a renewed and widespread resurgence.

Among many approaches, numerical techniques have been developed and exploited. For second order elliptic equations, a certainly not exhaustive list includes the multiscale finite element method [20], the residual-free bubble techniques [9], certain domain-decomposition techniques [27, 31], the two-grid techniques [30, 18], and a posteriori modeling techniques $[25,26]$. A scheme related directly to the work here is the variational multiscale finite element method [21, 22, 23]. Each scheme can be viewed as a subgrid technique in the sense that each attempts to resolve scales below the coarse grid scale by incorporating local computations into a global problem defined only on a coarse grid.

A new subgrid technique for upscaling an elliptic partial differential equation based on a certain combination of low order mixed finite elements was introduced in [5] and [1]. It involves the decomposition of the solution operator into two parts, one representing the coarse scale and the other representing the subgrid scale. The method is described in general terms, and numerical tests are given that demonstrate

${ }^{*}$ Received by the editors April 29, 2002; accepted for publication (in revised form) September 22, 2003; published electronically April 14, 2004. This work was supported by the U.S. National Science Foundation under grants DMS-9707015 and SBR-9873326.

http://www.siam.org/journals/sinum/42-2/40663.html

${ }^{\dagger}$ Department of Mathematics, University of Texas, 1 University Station C1200, Austin, TX 78712 and Institute for Computational Engineering and Sciences, University of Texas, 1 University Station C0200, Austin, TX 78712 (arbogast@ices.utexas.edu). 
the overall speed and convergence properties of the method in $[5,1]$. Applications to groundwater contaminant transport and petroleum simulation are given in [3, 4], wherein it is shown that the method has great potential to resolve fine-scale effects in practical problems. Complete details of implementation are presented in [2], as well as additional and more stringent numerical tests that apply the technique to two-phase porous medium problems with significant heterogeneity and wells. An advantage of this subgrid technique is that it needs no assumptions about the underlying physics. The data used in the simulation is to be provided directly on the fine scale.

The goals of this paper are threefold. First, we present a theoretical framework within which to understand the upscaling process. We achieve upscaling without the need for an explicit closure assumption or a restrictive assumption such as periodicity or the like. In general, it is difficult to analyze the errors introduced by a closure assumption; however, this problem does not arise here. Rather the ability of the upscaled model to capture fine-scale features in the solution becomes a question of approximation theory: how well do we approximate the upscaled model? Second, we generalize the mixed finite element technique of $[5,1]$ to essentially arbitrary choices of mixed spaces. Because of the upscaling framework, these methods can be implemented very efficiently and require the solution of a global problem defined only on the coarse grid. Finally, we provide an error analysis showing optimal order approximation.

Both an outline of the paper and a brief summary of results follow. After presenting in the next section the elliptic problem in mixed variational form posed in $W \times \mathbf{V} \subset L^{2} \times H$ (div), we then proceed in section 3 to define our framework within which we upscale the differential problem. We define the coarse grid we can ultimately compute over and use it to define a direct sum decomposition of $W \times \mathbf{V}$ into coarse and subgrid subspaces $W_{c} \times \mathbf{V}_{c}$ and $\delta W \times \delta \mathbf{V}$ such that (1) the divergence constraints $\nabla \cdot \mathbf{V}_{c}=W_{c}$ and $\nabla \cdot \delta \mathbf{V}=\delta W$, needed for local mass conservation over the coarse and subgrid scales, and (2) the space $\delta \mathbf{V}$ is locally supported over the coarse mesh, which is needed for upscaling the subgrid. This then leads to a decomposition of the variational problem into coarse and subgrid scale problems, with solutions in $W_{c} \times \mathbf{V}_{c}$ and $\delta W \times \delta \mathbf{V}$, although the two problems remain coupled.

We define in section 4 the $\delta$-solution operator as the solution of the subgrid problem. It is used to relate the subgrid to the coarse solution, and it is a well-defined operator that takes $W_{c} \times \mathbf{V}_{c}$ to $\delta W \times \delta \mathbf{V}$. Since this operator is localized in space, it can be used to control the fine scales. We use it in the coarse problem to remove direct reference to the subgrid, resulting in the upscaled problem involving only the coarse-scale solution.

In section 5 we exploit the two-scale structure of the solution to define an efficient mixed finite element method. We use any of the usual mixed elements to approximate the $\delta$-solution operator and also any choice of mixed spaces to approximate the upscaled coarse solution. This defines many families of two-scale, mixed spaces. Our approximation can be viewed as a type of variational multiscale method [21, 22] or a residual-free bubble technique [9]. A numerical Green's function approach makes the approximation to the subgrid operator efficient to compute.

In section 6 we analyze the approximation error. We show optimal order a priori error estimates. Care must be taken, as the two-scale decomposition depends on the coarse grid. We therefore analyze the combined system, showing approximation of the full solution. The key development here is the definition of a suitable mixed method $\pi$ operator that preserves the $L^{2}$-projection of the discrete divergence and approximates 
well in the two-scale context. Finally, in section 7 we apply the convergence theory to the special cases defined in [5] and [1].

2. A second order elliptic equation. Let $\Omega \subset \mathbb{R}^{n}, n=2$ or 3 , be a convex polygonal domain. Throughout the paper, for domain $\omega$, we denote by $L^{p}(\omega)$ the usual Lebesgue space of index $p, 1 \leq p \leq \infty$, and by $W^{k, p}(\omega)$ the usual Sobolev space of $k$ weak derivatives in $L^{p}(\omega)$. We denote by $(\cdot, \cdot)_{\omega}$ the $L^{2}(\omega)$-inner product (i.e., Lebesgue integration over $\omega$ ). Moreover, $\|\cdot\|_{k, \omega}$ is the norm of $H^{k}(\omega) \equiv W^{k, 2}(\omega)$. In the notation we may suppress $\omega$ when it is $\Omega$.

Decompose $\partial \Omega=\bar{\Gamma}_{N} \cup \bar{\Gamma}_{R}$, where $\Gamma_{N}$ and $\Gamma_{R}$ are disjoint open sets in $\partial \Omega$, and let $\nu$ be the outer unit normal vector. The problem is to find the unknown functions $p$ (pressure) and $\mathbf{u}$ (velocity) satisfying

$$
\begin{array}{ll}
a p+\nabla \cdot \mathbf{u}=b & \text { in } \Omega, \\
\mathbf{u}=-d(\nabla p-c) & \text { in } \Omega, \\
\mathbf{u} \cdot \nu=g_{N} & \text { on } \Gamma_{N}, \\
\alpha \mathbf{u} \cdot \nu=p-g_{R} & \text { on } \Gamma_{R},
\end{array}
$$

wherein $a \in L^{\infty}(\Omega)$ is nonnegative, $b \in L^{2}(\Omega), c \in\left(L^{2}(\Omega)\right)^{n}$, and $d$ is a second order uniformly positive definite symmetric tensor in $\left(L^{\infty}(\Omega)\right)^{n \times n}$ (i.e., $d$ and $d^{-1}$ are both uniformly elliptic and uniformly bounded). The boundary conditions represent Neumann and Robin (and Dirichlet, if $\alpha=0$ ) conditions for suitably nice functions $g_{N}, g_{R}$, and $\alpha \geq 0$. We assume that a unique and sufficiently regular solution to this system exists and that the coefficients are sufficiently regular for the error analysis to follow.

A special case arises if $a$ vanishes identically on all of $\Omega$ and $\Gamma_{N}=\partial \Omega$. Then it is well known and follows from the divergence theorem that solvability requires the compatibility condition

$$
\int_{\Omega} b(x) d x=\int_{\partial \Omega} g_{N}(x) d s(x) .
$$

In this case, we obtain $p$ only up to an arbitrary constant.

To enforce conservation of mass (2.1) locally, we base our method on a mixed variational formulation. Let

$$
H(\operatorname{div} ; \Omega)=\left\{\mathbf{v} \in\left(L^{2}(\Omega)\right)^{n}: \nabla \cdot \mathbf{v} \in L^{2}(\Omega)\right\}
$$

denote the usual space, with the inner product

$$
\left(\mathbf{v}_{1}, \mathbf{v}_{2}\right)_{H(\text { div })}=\left(\mathbf{v}_{1}, \mathbf{v}_{2}\right)+\left(\nabla \cdot \mathbf{v}_{1}, \nabla \cdot \mathbf{v}_{2}\right)
$$

and norm $\|\mathbf{v}\|_{H(\text { div })}=(\mathbf{v}, \mathbf{v})_{H(\text { div })}^{1 / 2}$, and let

$$
\mathbf{V}=\left\{\mathbf{v} \in H(\operatorname{div} ; \Omega): \mathbf{v} \cdot \nu=0 \text { on } \Gamma_{N}\right\},
$$

which is a closed subspace. To impose the Neumann boundary condition, we need to extend $g_{N}$ to some fixed vector $\mathbf{v}_{g_{N}} \in H(\operatorname{div} ; \Omega)$ such that

$$
\mathbf{v}_{g_{N}} \cdot \nu=g_{N} \text { on } \Gamma_{N} \quad \text { and } \quad \mathbf{v}_{g_{N}} \cdot \nu=0 \text { on } \Gamma_{R} .
$$

Finally, let $W=L^{2}(\Omega)$, or let $W=L^{2}(\Omega) / \mathbb{R}=\left\{w \in L^{2}(\Omega): \int_{\Omega} w(x) d x=0\right\}$ if $p$ will be defined only up to a constant. 
The mixed variational problem equivalent to (2.1)-(2.4) is to find $\mathbf{u} \in \mathbf{V}+\mathbf{v}_{g_{N}}$ and $p \in W$ such that

$$
\begin{array}{ll}
(a p, w)+(\nabla \cdot \mathbf{u}, w)=(b, w) & \forall w \in W \\
\left(d^{-1} \mathbf{u}, \mathbf{v}\right)+(\alpha \mathbf{u} \cdot \nu, \mathbf{v} \cdot \nu)_{\Gamma_{R}}-(p, \nabla \cdot \mathbf{v})=(c, \mathbf{v})-\left(g_{R}, \mathbf{v} \cdot \nu\right)_{\Gamma_{R}} & \forall \mathbf{v} \in \mathbf{V}
\end{array}
$$

Note that (2.3) is imposed as an essential condition and (2.4) is imposed weakly as a natural boundary condition.

3. Separation of scales. We recall that a Hilbert space $H$ is the direct sum of $M$ and $N$ if $H=M+N$ and $M$ and $N$ are closed subspaces that intersect only at the zero vector. We denote this fact by $H=M \oplus N$. In this case, given $x \in H$, there is some unique $m \in M$ and $n \in N$ such that $x=m+n$. We note the following result, which is an exercise in the application of the closed graph theorem [29].

Proposition 3.1. If $H$ is a Hilbert space and $H=M \oplus N$, then the operator $\tilde{\mathcal{P}}_{M}: H \rightarrow M$ defined for $x \in H$ by $\breve{\mathcal{P}}_{M} x=m$, where $x=m+n, m \in M$ and $n \in N$, is a bounded linear (but possibly nonorthogonal) projection.

We expand functions in $W \times \mathbf{V}$ uniquely according to a direct sum decomposition of the spaces. We base our decomposition on our two primary requirements: that the finer (i.e., "subgrid") scales be localized and that mass conservation is maintained. To do so we choose a coarse mesh partition $\mathcal{T}_{H}$ of $\Omega$ of a finite number of convex elements over which we will decompose the solution into coarse and local (i.e., "subgrid") pieces. The choice is mostly arbitrary at this point, but later the mesh will be used as the coarse mesh we compute on. We do, however, need a nondegeneracy condition. We assume that there is some universal fixed constant $\gamma>0$ such that any choice of $\mathcal{T}_{H}$ satisfies

$$
\operatorname{msr}\left(E_{c}\right) \geq \gamma\left(\operatorname{diam}\left(E_{c}\right)\right)^{n} \quad \forall E_{c} \in \mathcal{T}_{H},
$$

where $\operatorname{msr}\left(E_{c}\right)$ is the measure of $E_{c}$ and $\operatorname{diam}\left(E_{c}\right)$ is its diameter.

3.1. A two-scale decomposition of $\boldsymbol{W} \times \mathbf{V}$. As is well known, the divergence operator maps $\mathbf{V}$ onto $W$. The range of the divergence operator must be decomposed into a direct sum decomposition $W=W_{c} \oplus \delta W$ of closed subspaces. For our purposes, the decomposition is arbitrary, except that we must insist on two properties. First,

$$
\delta W \subset\left(W_{c}^{1}\right)^{\perp}, \quad W_{c}^{1}=\left\{w_{c} \in W: w_{c} \text { is constant } \forall \text { coarse elements } E_{c} \in \mathcal{T}_{H}\right\},
$$

with respect to the $L^{2}(\Omega)$-inner product.

Second, we insist that there is a uniformity in the separation of $W_{c}$ and $\delta W$. We define the possibly nonorthogonal projections

$$
\tilde{\mathcal{P}}_{W_{c}}: W \rightarrow W_{c} \quad \text { and } \quad \tilde{\mathcal{P}}_{\delta W}: W \rightarrow \delta W
$$

with respect to the direct sum decomposition. By Proposition 3.1, these operators are bounded but not necessarily uniformly so with respect to the coarse mesh $\mathcal{T}_{H}$ selected. Our requirement is that in fact these are bounded uniformly: there is some universal constant $C$, independent of the coarse mesh $\mathcal{T}_{H}$, such that

$$
\left\|w_{c}\right\|_{0}+\|\delta w\|_{0} \leq C\|w\|_{0},
$$

where $w=w_{c}+\delta w \in W_{c} \oplus \delta W$. We can easily achieve this property if, for example, $W_{c} \subset W$ such that $W_{c}^{1} \subset W_{c}$ is given arbitrarily and $\delta W=W_{c}^{\perp}$. However, we maintain flexibility by not assuming strict orthogonality. 
To obtain a decomposition of $\mathbf{V}$, we first define

$$
\begin{aligned}
& \mathbf{V}_{c}^{1}=\left\{\mathbf{v} \in \mathbf{V}: \nabla \cdot \mathbf{v} \in W_{c}\right\}, \\
& \delta \mathbf{V}^{1}=\left\{\delta \mathbf{v} \in \mathbf{V}: \nabla \cdot \delta \mathbf{v} \in \delta W \text { and } \delta \mathbf{v} \cdot \nu=0 \text { on } \partial E_{c} \forall E_{c} \in \mathcal{T}_{H}\right\}
\end{aligned}
$$

Proposition 3.2. It follows that

(a) $\mathbf{V}_{c}^{1}$ and $\delta \mathbf{V}^{1}$ are closed subspaces of $\mathbf{V}$;

(b) $\mathbf{V}=\mathbf{V}_{c}^{1}+\delta \mathbf{V}^{1}$;

(c) $\mathbf{V}_{c}^{1} \cap \delta \mathbf{V}^{1}=\left\{\mathbf{v} \in \mathbf{V}: \nabla \cdot \mathbf{v}=0\right.$ and $\mathbf{v} \cdot \nu=0$ on $\left.\partial E_{c} \forall E_{c} \in \mathcal{T}_{H}\right\}$;

(d) $\nabla \cdot \mathbf{V}_{c}^{1}=W_{c}$ and $\nabla \cdot \delta \mathbf{V}^{1}=\delta W$.

Proof. For (a), first note that each space is a linear subspace. The divergence operator is continuous on $\mathbf{V}$, so $\mathbf{V}_{c}^{1}$ is closed. Finally, we note that $\delta \mathbf{V}^{1}$ is the intersection of a closed subspace of $\mathbf{V}$ (the vectors $\delta \mathbf{v}$ with $\nabla \cdot \delta \mathbf{v} \in \delta W$ ) and the kernel of a finite number of normal trace operators, so $\delta \mathbf{V}^{1}$ is also closed.

To see (d), we consider an auxiliary elliptic problem. Given $\delta w \in \delta W$, on each $E_{c} \in \mathcal{T}_{H}$ let $\varphi \in H^{1}\left(E_{c}\right)$ solve the linear problem

$$
\begin{array}{ll}
\Delta \varphi=\delta w & \text { in } E_{c}, \\
\nabla \varphi \cdot \nu=0 & \text { on } \partial E_{c} .
\end{array}
$$

This problem is solvable because $\delta w \perp W_{c}^{1}$, so $\delta w$ satisfies the compatibility condition. Set $\delta \mathbf{v}=\nabla \varphi$. It is easy to conclude that $\delta \mathbf{v} \in \mathbf{V}$, since the normal traces match (in fact vanish) on each side of $\partial E_{c} \cap \Omega \forall E_{c} \in \mathcal{T}_{H}$. Thus we conclude that $\delta \mathbf{v} \in \delta \mathbf{V}^{1}$, and so $\delta W \subset \nabla \cdot \delta \mathbf{V}^{1}$. The opposite inclusion holds by definition, so $\nabla \cdot \delta \mathbf{V}^{1}=\delta W$. Similarly, given $w_{c} \in W_{c}$, let $\psi \in H^{1}(\Omega)$ solve the linear problem

$$
\begin{array}{ll}
\Delta \psi=w_{c} & \text { in } \Omega, \\
\nabla \psi \cdot \nu=0 & \text { on } \Gamma_{N}, \\
\psi=0 & \text { on } \Gamma_{R} .
\end{array}
$$

Then $\mathbf{v}_{c}=\nabla \psi \in \mathbf{V}_{c}^{1}$ allows us to conclude that $\nabla \cdot \mathbf{V}_{c}^{1}=W_{c}$.

For (b), we know that $\mathbf{V} \supset \mathbf{V}_{c}^{1}+\delta \mathbf{V}^{1}$, so consider any $\mathbf{v} \in \mathbf{V}$ and decompose $\nabla \cdot \mathbf{v}=w_{c}+\delta w$ for $w_{c} \in W_{c}$ and $\delta w \in \delta W$. Construct $\varphi$ and $\delta \mathbf{v}=\nabla \varphi$ from $\delta w$ as in (3.4)-(3.5) above. Then we conclude that $\mathbf{v}_{c}=\mathbf{v}-\delta \mathbf{v} \in \mathbf{V}_{c}^{1}$, and so $\mathbf{V} \subset \mathbf{V}_{c}^{1}+\delta \mathbf{V}^{1}$. Finally, (c) follows trivially from (d), since $W_{c} \cap \delta W=\{0\}$.

The proof above suggests the following Helmholtz decomposition. Let

$$
\begin{aligned}
\mathbf{V}_{c}^{p} & =\left\{\mathbf{v}_{c}^{p} \in \mathbf{V}_{c}^{1}: \mathbf{v}_{c}^{p}=\nabla \psi \text { for some } w_{c} \in W_{c} \text { and } \psi \text { satisfying }(3.6)-(3.8)\right\} \\
\mathbf{V}^{s} & =\left\{\mathbf{v}^{s} \in \mathbf{V}: \nabla \cdot \mathbf{v}^{s}=0\right\} \subset \mathbf{V}_{c}^{1} .
\end{aligned}
$$

These spaces are clearly closed subspaces, and we claim that $\mathbf{V}_{c}^{p} \cap \mathbf{V}^{s}=\{0\}$. Let $\mathbf{v}$ be a member of both. Then there is some scalar potential function $\psi$ such that $\mathbf{v}=\nabla \psi$, and $\nabla \cdot \mathbf{v}=\Delta \psi=0$. Moreover, the boundary conditions from $\mathbf{V}_{c}^{p}$ imply that $\psi$ is constant (zero if $\Gamma_{R} \neq \emptyset$ ), and so $\mathbf{v}=0$. Thus we conclude that in fact

$$
\mathbf{V}_{c}^{1}=\mathbf{V}_{c}^{p} \oplus \mathbf{V}^{s}
$$

is a direct sum of potential and solenoidal vector fields. Similarly, we have the closed subspace

$$
\delta \mathbf{V}^{p}=\left\{\delta \mathbf{v}^{p} \in \delta \mathbf{V}^{1}: \delta \mathbf{v}^{p}=\nabla \varphi \text { for some } \delta w \in \delta W \text { and } \varphi \text { satisfying (3.4)-(3.5) }\right\}
$$


and the direct sum

$$
\delta \mathbf{V}^{1}=\delta \mathbf{V}^{p} \oplus \delta \mathbf{V}^{s}, \quad \text { where } \quad \delta \mathbf{V}^{s}=\mathbf{V}^{s} \cap \delta \mathbf{V}^{1} .
$$

By similar reasoning, we conclude from $W_{c} \cap \delta W=\{0\}$ that $\mathbf{V}_{c}^{p} \cap \delta \mathbf{V}^{p}=\{0\}$, and thus

$$
\mathbf{V}=\mathbf{V}_{c}^{p} \oplus \delta \mathbf{V}^{p} \oplus \mathbf{V}^{s}
$$

TheOREM 3.3. There is some constant $C$, independent of $\mathcal{T}_{H}$, and there exist closed subspaces $\mathbf{V}_{c}$ and $\delta \mathbf{V}$ of $\mathbf{V}$ such that

(a) $\mathbf{V}=\mathbf{V}_{c} \oplus \delta \mathbf{V}$;

(b) $\nabla \cdot \mathbf{V}_{c}=W_{c}$ and $\nabla \cdot \delta \mathbf{V}=\delta W$;

(c) $\delta \mathbf{V} \subset \delta \mathbf{V}^{1}=\left\{\delta \mathbf{v} \in \mathbf{V}: \nabla \cdot \delta \mathbf{v} \in \delta W\right.$ and $\delta \mathbf{v} \cdot \nu=0$ on $\left.\partial E_{c} \forall E_{c} \in \mathcal{T}_{H}\right\}$;

(d) for $\mathbf{v}=\mathbf{v}_{c}+\delta \mathbf{v} \in \mathbf{V}_{c} \oplus \delta \mathbf{V}$ given,

$$
\left\|\mathbf{v}_{c}\right\|_{H(d i v)}+\|\delta \mathbf{v}\|_{H(d i v)} \leq C\|\mathbf{v}\|_{H(d i v)} .
$$

Moreover, a choice exists such that also the potential vector fields

(e) $\mathbf{V}_{c}^{p} \subset \mathbf{V}_{c}$ and $\delta \mathbf{V}^{p} \subset \delta \mathbf{V}$.

That is, (d) says that the projection operators defined by the direct sum,

$$
\tilde{\mathcal{P}}_{\mathbf{V}_{c}}: \mathbf{V} \rightarrow \mathbf{V}_{c} \quad \text { and } \quad \tilde{\mathcal{P}}_{\delta \mathbf{V}}: \mathbf{V} \rightarrow \delta \mathbf{V},
$$

are bounded independently of $\mathcal{T}_{H}$.

Proof. The troublesome part of (3.9) are the solenoidal fields $\mathbf{V}^{s}$. With respect to the $H$ (div)-inner product, let

$$
\mathbf{V}_{c}^{s}=\left(\delta \mathbf{V}^{s}\right)^{\perp} \cap \mathbf{V}^{s}=\left\{\mathbf{v}_{c}^{s} \in \mathbf{V}^{s}: \mathbf{v}_{c}^{s} \perp \delta \mathbf{V}^{s}\right\} .
$$

Then $\mathbf{V}^{s}=\mathbf{V}_{c}^{s} \oplus \delta \mathbf{V}^{s}$, and we can define

$$
\mathbf{V}_{c}=\mathbf{V}_{c}^{p} \oplus \mathbf{V}_{c}^{s} \quad \text { and } \quad \delta \mathbf{V}=\delta \mathbf{V}^{p} \oplus \delta \mathbf{V}^{s}
$$

satisfying (a)-(c) and (e).

We need to examine the construction more carefully to conclude (d). Let $\mathbf{v} \in \mathbf{V}$ be given, and decompose

$$
\nabla \cdot \mathbf{v}=w_{c}+\delta w=\tilde{\mathcal{P}}_{W_{c}} \nabla \cdot \mathbf{v}+\tilde{\mathcal{P}}_{\delta W} \nabla \cdot \mathbf{v} \in W_{c} \oplus \delta W .
$$

We then construct $\delta \mathbf{v}^{p}=\nabla \varphi \in \delta \mathbf{V}^{p}$ from (3.4)-(3.5) using the given $\delta w$ and note that standard elliptic energy estimates show that on each $E_{c} \in \mathcal{T}_{H}$,

$$
\begin{aligned}
\left\|\delta \mathbf{v}^{p}\right\|_{0, E_{c}}^{2} & =\|\nabla \varphi\|_{0, E_{c}}^{2}=\left(\tilde{\mathcal{P}}_{\delta W} \nabla \cdot \mathbf{v}, \varphi\right)_{E_{c}} \\
& \leq\left\|\tilde{\mathcal{P}}_{\delta W} \nabla \cdot \mathbf{v}\right\|_{0, E_{c}}\|\varphi\|_{0, E_{c}} \leq C\left\|\tilde{\mathcal{P}}_{\delta W} \nabla \cdot \mathbf{v}\right\|_{0, E_{c}}\|\nabla \varphi\|_{0, E_{c}},
\end{aligned}
$$

where $C$ is the Poincaré inequality constant [17] for $E_{c}$, which is proportional to $\frac{\operatorname{diam}\left(E_{c}\right)^{n}}{\operatorname{msr}\left(E_{c}\right)^{1-1 / n}}$ and therefore universally bounded by the nondegeneracy assumption (3.1). Thus, from (3.3),

$$
\left\|\delta \mathbf{v}^{p}\right\|_{H(\text { div })} \leq C\left\|\tilde{\mathcal{P}}_{\delta W} \nabla \cdot \mathbf{v}\right\|_{0} \leq C\|\mathbf{v}\|_{H(\text { div })} .
$$


Similarly, we construct $\mathbf{v}_{c}^{p}=\nabla \psi \in \mathbf{V}_{c}^{p}$ from (3.6)-(3.8) using the given $w_{c}$ and conclude that

$$
\left\|\mathbf{v}_{c}^{p}\right\|_{H(\text { div })} \leq C\|\mathbf{v}\|_{H(\text { div })} .
$$

Now $\mathbf{v}=\mathbf{v}_{c}^{p}+\delta \mathbf{v}^{p}+\mathbf{v}^{s} \in \mathbf{V}_{c}^{p} \oplus \delta \mathbf{V}^{p} \oplus \mathbf{V}^{s}$ by (3.9), and $\mathbf{v}^{s}=\mathbf{v}_{c}^{s}+\delta \mathbf{v}^{s} \in \mathbf{V}_{c}^{s} \oplus \delta \mathbf{V}^{s}$, which is an orthogonal decomposition, so

$$
\left\|\mathbf{v}_{c}^{s}\right\|_{H(\text { div })}^{2}+\left\|\delta \mathbf{v}^{s}\right\|_{H(\text { div })}^{2}=\left\|\mathbf{v}^{s}\right\|_{H(\text { div })}^{2}=\left\|\mathbf{v}-\mathbf{v}_{c}^{p}-\delta \mathbf{v}^{p}\right\|_{H(\text { div })}^{2} \leq C\|\mathbf{v}\|_{H(\text { div })}^{2} .
$$

Finally,

$$
\begin{aligned}
& \left\|\mathbf{v}_{c}\right\|_{H(\text { div })}=\left\|\mathbf{v}_{c}^{p}+\mathbf{v}_{c}^{s}\right\|_{H(\text { div })} \leq C\|\mathbf{v}\|_{H(\text { div })}, \\
& \|\delta \mathbf{v}\|_{H(\text { div })}=\left\|\delta \mathbf{v}^{p}+\delta \mathbf{v}^{s}\right\|_{H(\text { div })} \leq C\|\delta \mathbf{v}\|_{H(\text { div })},
\end{aligned}
$$

and the proof is complete.

Thus (a) gives us a unique decomposition of vectors in $\mathbf{V}$, (b) allows us to enforce mass conservation over $\mathcal{T}_{H}$ on both the coarse and subgrid scales, (c) gives us a locality property of the space $\delta \mathbf{V}$ that we can exploit later, and (d) gives us a uniformity property of the decomposition independent of $\mathcal{T}_{H}$. The specific choice of decomposition appears to be unimportant for our purposes, although we will revisit this question later in section 5.1. In what follows, we fix a choice of $\mathbf{V}_{c}$ and $\delta \mathbf{V}$ satisfying the properties (a)-(d) of the theorem.

3.2. Separation of scales in the equations. Recall that $\mathbf{v}_{g_{N}} \in H(\operatorname{div} ; \Omega)$ satisfies the Neumann boundary condition. Decompose the solution

$$
\begin{aligned}
& p=p_{c}+\delta p \in W_{c} \oplus \delta W, \\
& \mathbf{u}=\mathbf{u}_{c}+\delta \mathbf{u}+\mathbf{v}_{g_{N}} \in \mathbf{V}_{c} \oplus \delta \mathbf{V}+\mathbf{v}_{g_{N}} .
\end{aligned}
$$

Then we decompose (2.6)-(2.7) by choosing test functions restricted to the spaces $W_{c} \times \mathbf{V}_{c}$ or $\delta W \times \delta \mathbf{V}$. This results in an equivalent system of the four equations (3.12)-(3.15) below. For convenience, let

$$
\begin{aligned}
& b^{*}=b-\nabla \cdot \mathbf{v}_{g_{N}}, \\
& c^{*}=c-d^{-1} \mathbf{v}_{g_{N}} .
\end{aligned}
$$

Coarse-scale equations. Find $\mathbf{u}_{c} \in \mathbf{V}_{c}$ and $p_{c} \in W_{c}$ such that

$$
\begin{array}{ll}
\left(a\left(p_{c}+\delta p\right), w_{c}\right)+\left(\nabla \cdot\left(\mathbf{u}_{c}+\delta \mathbf{u}\right), w_{c}\right)=\left(b^{*}, w_{c}\right) & \forall w_{c} \in W_{c} \\
\left(d^{-1}\left(\mathbf{u}_{c}+\delta \mathbf{u}\right), \mathbf{v}_{c}\right)+\left(\alpha \mathbf{u}_{c} \cdot \nu, \mathbf{v}_{c} \cdot \nu\right)_{\Gamma_{R}}-\left(p_{c}+\delta p, \nabla \cdot \mathbf{v}_{c}\right) & \\
\quad=\left(c^{*}, \mathbf{v}_{c}\right)-\left(g_{R}, \mathbf{v}_{c} \cdot \nu\right)_{\Gamma_{R}} & \forall \mathbf{v}_{c} \in \mathbf{V}_{c} .
\end{array}
$$

Subgrid $\delta$-scale equations. Find $\delta \mathbf{u} \in \delta \mathbf{V}$ and $\delta p \in \delta W$ such that

$$
\begin{array}{ll}
\left(a\left(p_{c}+\delta p\right), \delta w\right)+\left(\nabla \cdot\left(\mathbf{u}_{c}+\delta \mathbf{u}\right), \delta w\right)=\left(b^{*}, \delta w\right) & \forall \delta w \in \delta W \\
\left(d^{-1}\left(\mathbf{u}_{c}+\delta \mathbf{u}\right), \delta \mathbf{v}\right)-\left(p_{c}+\delta p, \nabla \cdot \delta \mathbf{v}\right)=\left(c^{*}, \delta \mathbf{v}\right) & \forall \delta \mathbf{v} \in \delta \mathbf{V} .
\end{array}
$$

4. The $\boldsymbol{\delta}$-solution operator and upscaling. The systems (3.12)-(3.13) and (3.14)-(3.15) are coupled together, and as written they do not allow us to exploit the locality of $\delta \mathbf{V}$. Our goal now is to rewrite (3.12)-(3.13) independently of $\delta p$ and $\delta \mathbf{u}$. To do so, we need to write these quantities in terms of $p_{c}$ and $\mathbf{u}_{c}$. 


\subsection{Solvability of the subgrid scale equations.}

Lemma 4.1. Given $\left(p_{c}, \mathbf{u}_{c}\right) \in W_{c} \times \mathbf{V}_{c}$, there exists a unique solution $(\delta p, \delta \mathbf{u}) \in$ $\delta W \times \delta \mathbf{V}$ to (3.14)-(3.15). Moreover, there is some constant $C$, independent of the coarse mesh $\mathcal{T}_{H}$ and the specific decomposition of $W \times \mathbf{V}$ selected, such that

$$
\|\delta p\|_{0}+\|\delta \mathbf{u}\|_{H(d i v)} \leq C\left\{\|b\|_{0}+\|c\|_{0}+\left\|\mathbf{v}_{g_{N}}\right\|_{H(d i v)}+\left\|p_{c}\right\|_{0}+\left\|\mathbf{u}_{c}\right\|_{H(d i v)}\right\} .
$$

We can prove Lemma 4.1 using the theory of saddle point problems $[6,8,13,7]$. We need a generalization of the theory developed in, e.g., [13]. Consider the following abstract problem: Find $\check{p} \in \check{W}$ and $\check{\mathbf{u}} \in \check{\mathbf{V}}$ such that

$$
\begin{array}{ll}
\check{c}(\check{p}, \check{w})+(\nabla \cdot \check{\mathbf{u}}, \check{w})=F(\check{w}) & \forall \check{w} \in \check{W}, \\
\check{a}(\check{\mathbf{u}}, \check{\mathbf{v}})-(\check{p}, \nabla \cdot \check{\mathbf{v}})=G(\check{\mathbf{v}}) & \forall \check{\mathbf{v}} \in \check{\mathbf{V}},
\end{array}
$$

where $\check{W} \subset L^{2}(\Omega)$ and $\check{\mathbf{V}} \subset H(\operatorname{div} ; \Omega)$ are Hilbert spaces. The following result is a simple corollary of the more general theory [13, pp. 44-47].

TheOREM 4.2. Suppose that $\check{W} \subset L^{2}(\Omega)$ and $\check{\mathbf{V}} \subset H(\operatorname{div} ; \Omega)$ are Hilbert spaces such that $\nabla \cdot \check{\mathbf{V}}=\check{W}$. Suppose that $\check{a}$ and $\check{c}$ are continuous, symmetric, positive semidefinite bilinear forms on $\check{\mathbf{V}} \times \check{\mathbf{V}}$ and $\check{W} \times \check{W}$, respectively, and that $\check{a}$ is coercive on $\check{\mathbf{V}} \cap \operatorname{ker}(\nabla \cdot), G \in \check{\mathbf{V}}^{\prime}, F \in \check{W}^{\prime}$, and there exists $\beta>0$ such that

$$
\inf _{\check{w} \in \check{W}} \sup _{\check{\mathbf{v}} \in \check{\mathbf{V}}} \frac{(\nabla \cdot \check{\mathbf{v}}, \check{w})}{\|\check{\mathbf{v}}\|_{H(d i v)}\|\check{w}\|_{0}} \geq \beta>0 .
$$

Then there exists a unique solution $(\check{p}, \check{\mathbf{u}}) \in \check{W} \times \check{\mathbf{V}}$ to (4.1)-(4.2), and there is a constant $C$ such that

$$
\|\check{p}\|_{0}+\|\check{\mathbf{u}}\|_{H(\text { div })} \leq C\{\|F\|+\|G\|\}
$$

where $C$ is a nonlinear function of $\|\check{a}\|$, $\|\check{c}\|$, the reciprocal of the coercivity bound for $\check{a}$, and $1 / \beta$ that is bounded on bounded subsets.

The key result is to prove the celebrated inf-sup condition (4.3). This condition is known to hold over $W \times \mathbf{V}$, and the following corollary is well known and uses the fact that for $\mathbf{v} \in \mathbf{V}$,

$$
\left(g_{R}, \mathbf{v} \cdot \nu\right)_{\Gamma_{R}}=\left(g_{R}, \mathbf{v} \cdot \nu\right)_{\partial \Omega} \leq C\left\|g_{R}\right\|_{1 / 2, \partial \Omega}\|\mathbf{v}\|_{H(\operatorname{div})},
$$

where $g_{R}$ on $\partial \Omega$ is any fixed bounded extension.

COROLlary 4.3. There exists a unique solution to (2.6)-(2.7), and there is some constant $C$ depending on $a, d$, and the inf-sup bound such that

$$
\|p\|_{0}+\|\mathbf{u}\|_{H(d i v)} \leq C\left\{\|b\|_{0}+\|c\|_{0}+\left\|\mathbf{v}_{g_{N}}\right\|_{H(d i v)}+\left\|g_{R}\right\|_{1 / 2, \partial \Omega}\right\} .
$$

LEMma 4.4. The inf-sup condition holds over both $W_{c} \times \mathbf{V}_{c}$ and $\delta W \times \delta \mathbf{V}$, with constants independent of the coarse mesh $\mathcal{T}_{H}$ and the specific decomposition of $W \times \mathbf{V}$ selected.

Proof. For $W_{c} \times \mathbf{V}_{c}$, we have the following argument. Given $w_{c} \in W_{c}$, solve for $\psi \in H^{1}(\Omega)$ satisfying (3.6)-(3.8), with $\int_{\Omega} \psi d x=0$ if $\Gamma_{N}=\partial \Omega$. Set $\mathbf{v}=\nabla \psi$. Then

$$
\|\mathbf{v}\|_{0}^{2}=\|\nabla \psi\|_{0}^{2}=\left(w_{c}, \psi\right) \leq\left\|w_{c}\right\|_{0}\|\psi\|_{0} \leq C\left\|w_{c}\right\|_{0}\|\nabla \psi\|_{0},
$$


by Poincaré's inequality [17], so $\|\mathbf{v}\|_{H(\text { div) }} \leq C\left\|w_{c}\right\|_{0}$. Let $\mathbf{v}=\hat{\mathbf{v}}_{c}+\delta \mathbf{v}$, where $\hat{\mathbf{v}}_{c} \in \mathbf{V}_{c}$ and $\delta \mathbf{v} \in \delta \mathbf{V}$. We note that $w_{c}=\nabla \cdot \mathbf{v}=\nabla \cdot \hat{\mathbf{v}}_{c} \in W_{c}$. Moreover,

$$
\left\|\hat{\mathbf{v}}_{c}\right\|_{H(\text { div })}=\left\|\tilde{\mathcal{P}}_{\mathbf{V}_{c}} \mathbf{v}\right\|_{H(\text { div })} \leq C\|\mathbf{v}\|_{H(\text { div })} \leq C\left\|w_{c}\right\|_{0}
$$

Thus

$$
\begin{aligned}
\inf _{w_{c} \in W_{c}} \sup _{c} \in \frac{\left(\nabla \cdot \mathbf{v}_{c}, w_{c}\right)}{\left\|\mathbf{v}_{c}\right\|_{H(\text { div })}\left\|w_{c}\right\|_{0}} & \geq \inf _{w_{c} \in W_{c}} \frac{\left(\nabla \cdot \hat{\mathbf{v}}_{c}, w_{c}\right)}{\left\|\hat{\mathbf{v}}_{c}\right\|_{H(\text { div })}\left\|w_{c}\right\|_{0}} \\
& =\inf _{w_{c} \in W_{c}} \frac{\left\|w_{c}\right\|_{0}}{\left\|\hat{\mathbf{v}}_{c}\right\|_{H(\text { div })}} \geq \frac{1}{C}>0 .
\end{aligned}
$$

The proof for $\delta W \times \delta \mathbf{V}$ is entirely similar and omitted.

Proof of Lemma 4.1. We can rewrite the subgrid $\delta$-scale equations (3.14)-(3.15) in the form of the abstract problem (4.1)-(4.2) by taking $\check{\mathbf{V}}=\delta \mathbf{V}$ and $\breve{W}=\delta W$ and by defining

$$
\begin{aligned}
& \check{c}\left(\delta w_{1}, \delta w_{2}\right)=\left(a \delta w_{1}, \delta w_{2}\right), \\
& \check{a}\left(\delta \mathbf{v}_{1}, \delta \mathbf{v}_{2}\right)=\left(d^{-1} \delta \mathbf{v}_{1}, \delta \mathbf{v}_{2}\right), \\
& F(\delta w)=\left(b^{*}-a p_{c}-\nabla \cdot \mathbf{u}_{c}, \delta w\right), \\
& G(\delta \mathbf{v})=\left(c^{*}-d^{-1} \mathbf{u}_{c}, \delta \mathbf{v}\right)+\left(p_{c}, \nabla \cdot \delta \mathbf{v}\right) .
\end{aligned}
$$

Easily, the bilinear forms $\check{a}$ and $\check{c}$ are continuous, symmetric, and nonnegative on $\delta \mathbf{V}$, and $\check{a}$ is coercive on $\delta \mathbf{V} \cap \operatorname{ker}(\nabla \cdot)$, with constants depending on the coefficients $a, c$, and $d$. Moreover, $F$ and $G$ are continuous linear functionals. Lemma 4.4 gives us the inf-sup condition (4.3), so the hypotheses required by Theorem 4.2 are satisfied by the system, and so the conclusions follow.

4.2. The $\delta$-solution operator. Lemma 4.1 allows us to define the solution operator of the subgrid $\delta$-scale equations (3.14)-(3.15) in terms of the coarse-scale solution. It is in fact an affine operator with constant and linear parts.

Constant part of the $\delta$-solution operator. Find $\delta \bar{p} \in \delta W$ and $\delta \overline{\mathbf{u}} \in \delta \mathbf{V}$ such that

$$
\begin{array}{ll}
(a \delta \bar{p}, \delta w)+(\nabla \cdot \delta \overline{\mathbf{u}}, \delta w)=\left(b^{*}, \delta w\right) & \forall \delta w \in \delta W \\
\left(d^{-1} \delta \overline{\mathbf{u}}, \delta \mathbf{v}\right)-(\delta \bar{p}, \nabla \cdot \delta \mathbf{v})=\left(c^{*}, \delta \mathbf{v}\right) & \forall \delta \mathbf{v} \in \delta \mathbf{V} .
\end{array}
$$

$W_{c}$-linear part of the $\delta$-solution operator. For $w_{c} \in W_{c}$, find $\delta \tilde{p} \in \delta W$ and $\delta \tilde{\mathbf{u}} \in \delta \mathbf{V}$ such that

$$
\begin{array}{ll}
\left(a\left(w_{c}+\delta \tilde{p}\right), \delta w\right)+(\nabla \cdot \delta \tilde{\mathbf{u}}, \delta w)=0 & \forall \delta w \in \delta W \\
\left(d^{-1} \delta \tilde{\mathbf{u}}, \delta \mathbf{v}\right)-\left(w_{c}+\delta \tilde{p}, \nabla \cdot \delta \mathbf{v}\right)=0 & \forall \delta \mathbf{v} \in \delta \mathbf{V}
\end{array}
$$

$\mathbf{V}_{c}$-linear part of the $\delta$-solution operator. For $\mathbf{v}_{c} \in \mathbf{V}_{c}$, find $\delta \hat{p} \in \delta W$ and $\delta \hat{\mathbf{u}} \in \delta \mathbf{V}$ such that

$$
\begin{array}{ll}
(a \delta \hat{p}, \delta w)+\left(\nabla \cdot\left(\mathbf{v}_{c}+\delta \hat{\mathbf{u}}\right), \delta w\right)=0 & \forall \delta w \in \delta W \\
\left(d^{-1}\left(\mathbf{v}_{c}+\delta \hat{\mathbf{u}}\right), \delta \mathbf{v}\right)-(\delta \hat{p}, \nabla \cdot \delta \mathbf{v})=0 & \forall \delta \mathbf{v} \in \delta \mathbf{V}
\end{array}
$$

The theory of saddle point problems allows us to conclude the solvability and boundedness of each system, so we have the following result. 
THEOREM 4.5. There exist bounded linear operators

$$
\begin{array}{lll}
\delta \tilde{p}: W_{c} \rightarrow \delta W & \text { and } & \delta \tilde{\mathbf{u}}: W_{c} \rightarrow \delta \mathbf{V}, \\
\delta \hat{p}: \mathbf{V}_{c} \rightarrow \delta W & \text { and } & \delta \hat{\mathbf{u}}: \mathbf{V}_{c} \rightarrow \delta \mathbf{V},
\end{array}
$$

bounded independent of the coarse mesh $\mathcal{T}_{H}$ and the specific decomposition of $W \times \mathbf{V}$ selected, defined by (4.6)-(4.9), and functions $\delta \bar{p} \in \delta W$ and $\delta \overline{\mathbf{u}} \in \delta \mathbf{V}$ defined by (4.4)-(4.5) such that

$$
\begin{aligned}
& \delta p=\delta \tilde{p}\left(p_{c}\right)+\delta \hat{p}\left(\mathbf{u}_{c}\right)+\delta \bar{p} \\
& \delta \mathbf{u}=\delta \tilde{\mathbf{u}}\left(p_{c}\right)+\delta \hat{\mathbf{u}}\left(\mathbf{u}_{c}\right)+\delta \overline{\mathbf{u}}
\end{aligned}
$$

Moreover, there is some constant $C$ such that

$$
\|\delta \bar{p}\|_{0}+\|\delta \overline{\mathbf{u}}\|_{H(d i v)} \leq C\left\{\|b\|_{0}+\|c\|_{0}+\left\|\mathbf{v}_{g_{N}}\right\|_{H(d i v)}\right\} .
$$

Because $\delta \mathbf{V} \cdot \nu=0$ on each $\partial E_{c}$ for $E_{c} \in \mathcal{T}_{H}, \delta \tilde{p}, \delta \tilde{\mathbf{u}}, \delta \hat{p}$, and $\delta \hat{\mathbf{u}}$ are locally defined operators. That is, the restriction to $E_{c}$ of the result is given by evaluating the restricted operators, which are defined by restricting the integrals to $E_{c}$ in (4.6)(4.9). Symbolically, we might write

$$
\begin{aligned}
\left.\delta \tilde{p}\left(p_{c}\right)\right|_{E_{c}}=\left.\delta \tilde{p}\right|_{E_{c}}\left(\left.p_{c}\right|_{E_{c}}\right) & \text { and }\left.\quad \delta \tilde{\mathbf{u}}\left(p_{c}\right)\right|_{E_{c}}=\left.\delta \tilde{\mathbf{u}}\right|_{E_{c}}\left(\left.p_{c}\right|_{E_{c}}\right), \\
\left.\delta \hat{p}\left(\mathbf{u}_{c}\right)\right|_{E_{c}}=\left.\delta \hat{p}\right|_{E_{c}}\left(\left.\mathbf{u}_{c}\right|_{E_{c}}\right) & \text { and }\left.\quad \delta \hat{\mathbf{u}}\left(\mathbf{u}_{c}\right)\right|_{E_{c}}=\left.\delta \hat{\mathbf{u}}\right|_{E_{c}}\left(\left.\mathbf{u}_{c}\right|_{E_{c}}\right)
\end{aligned}
$$

These operators are well defined, linear, and bounded uniformly with respect to $\mathcal{T}_{H}$ and the decomposition of $W \times \mathbf{V}$ selected.

In upscaling theory, results like Theorem 4.5 allow one to close the equations. That is, the fine scale is represented as an operator of the coarse scale. However, usually such a result is either assumed or additional assumptions are added to restrict the nature of the problem (such as assuming some kind of periodicity or ergodicity). Hence such results are often called closure assumptions. We have closed our system without the need of any additional assumptions.

4.3. The upscaled equation. If we substitute the $\delta$-solution operator into the coarse-scale equations (3.12)-(3.13), we obtain the following problem.

Asymmetric upscaled equations. Find $p_{c} \in W_{c}$ and $\mathbf{u}_{c} \in \mathbf{V}_{c}$ such that

$$
\begin{array}{rlr}
\left(a\left(p_{c}+\delta \tilde{p}\left(p_{c}\right)+\delta \hat{p}\left(\mathbf{u}_{c}\right)\right), w_{c}\right) & \\
\quad+\left(\nabla \cdot\left(\mathbf{u}_{c}+\delta \tilde{\mathbf{u}}\left(p_{c}\right)+\delta \hat{\mathbf{u}}\left(\mathbf{u}_{c}\right)\right), w_{c}\right) & \\
\quad( & \left(b^{*}-a \delta \bar{p}-\nabla \cdot \delta \overline{\mathbf{u}}, w_{c}\right) & \\
\left(d^{-1}\left(\mathbf{u}_{c}+\delta \tilde{\mathbf{u}}\left(p_{c}\right)+\delta \hat{\mathbf{u}}\left(\mathbf{u}_{c}\right)\right), \mathbf{v}_{c}\right) & \\
\quad+\left(\alpha \mathbf{u}_{c} \cdot \nu, \mathbf{v}_{c} \cdot \nu\right)_{\Gamma_{R}}-\left(p_{c}+\delta \tilde{p}\left(p_{c}\right)+\delta \hat{p}\left(\mathbf{u}_{c}\right), \nabla \cdot \mathbf{v}_{c}\right) & \\
\quad=\left(c^{*}-d^{-1} \delta \overline{\mathbf{u}}, \mathbf{v}_{c}\right)+\left(\delta \bar{p}, \nabla \cdot \mathbf{v}_{c}\right)-\left(g_{R}, \mathbf{v}_{c} \cdot \nu\right)_{\Gamma_{R}} & \forall \mathbf{v}_{c} \in \mathbf{V}_{c} .
\end{array}
$$

This system is posed entirely with respect to coarse-scale functions, so we say that it has been upscaled from the fine scale. However, this system is not symmetric, even though the original fine-scale system is symmetric. We can remedy this by noting several equivalences. First, note that from (4.6) and then (4.7),

$$
\begin{aligned}
\left(a\left(p_{c}+\delta \tilde{p}\left(p_{c}\right)\right), \delta \tilde{p}\left(w_{c}\right)\right) & =-\left(\nabla \cdot \delta \tilde{\mathbf{u}}\left(p_{c}\right), \delta \tilde{p}\left(w_{c}\right)\right) \\
& =-\left(d^{-1} \delta \tilde{\mathbf{u}}\left(w_{c}\right), \delta \tilde{\mathbf{u}}\left(p_{c}\right)\right)+\left(w_{c}, \nabla \cdot \delta \tilde{\mathbf{u}}\left(p_{c}\right)\right)
\end{aligned}
$$


and similarly from (4.9) and then (4.8),

$$
\begin{aligned}
\left(d^{-1}\left(\mathbf{u}_{c}+\delta \hat{\mathbf{u}}\left(\mathbf{u}_{c}\right)\right), \delta \hat{\mathbf{u}}\left(\mathbf{v}_{c}\right)\right) & =\left(\delta \hat{p}\left(\mathbf{u}_{c}\right), \nabla \cdot \delta \hat{\mathbf{u}}\left(\mathbf{v}_{c}\right)\right) \\
& =-\left(a \delta \hat{p}\left(\mathbf{v}_{c}\right), \delta \hat{p}\left(\mathbf{u}_{c}\right)\right)-\left(\nabla \cdot \mathbf{v}_{c}, \delta \hat{p}\left(\mathbf{u}_{c}\right)\right) .
\end{aligned}
$$

We also apply (4.9), (4.6), (4.7), and then (4.8) to obtain

$$
\begin{aligned}
\left(d^{-1} \delta \tilde{\mathbf{u}}\left(p_{c}\right), \mathbf{v}_{c}\right) & =\left(\delta \hat{p}\left(\mathbf{v}_{c}\right), \nabla \cdot \delta \tilde{\mathbf{u}}\left(p_{c}\right)\right)-\left(d^{-1} \delta \hat{\mathbf{u}}\left(\mathbf{v}_{c}\right), \delta \tilde{\mathbf{u}}\left(p_{c}\right)\right) \\
& =-\left(a\left(p_{c}+\delta \tilde{p}\left(p_{c}\right)\right), \delta \hat{p}\left(\mathbf{v}_{c}\right)\right)-\left(d^{-1} \delta \hat{\mathbf{u}}\left(\mathbf{v}_{c}\right), \delta \tilde{\mathbf{u}}\left(p_{c}\right)\right) \\
& =-\left(a\left(p_{c}+\delta \tilde{p}\left(p_{c}\right)\right), \delta \hat{p}\left(\mathbf{v}_{c}\right)\right)-\left(p_{c}+\delta \tilde{p}\left(p_{c}\right), \nabla \cdot \delta \hat{\mathbf{u}}\left(\mathbf{v}_{c}\right)\right) \\
& =-\left(a p_{c}, \delta \hat{p}\left(\mathbf{v}_{c}\right)\right)-\left(p_{c}, \nabla \cdot \delta \hat{\mathbf{u}}\left(\mathbf{v}_{c}\right)\right)+\left(\nabla \cdot \mathbf{v}_{c}, \delta \tilde{p}\left(p_{c}\right)\right) .
\end{aligned}
$$

Combining, we obtain a symmetric form for our system.

Symmetric upscaled equations. Find $p_{c} \in W_{c}$ and $\mathbf{u}_{c} \in \mathbf{V}_{c}$ such that

$$
\begin{aligned}
& \left(a\left(p_{c}+\delta \tilde{p}\left(p_{c}\right)\right), w_{c}+\delta \tilde{p}\left(w_{c}\right)\right)+\left(d^{-1} \delta \tilde{\mathbf{u}}\left(p_{c}\right), \delta \tilde{\mathbf{u}}\left(w_{c}\right)\right) \\
& +\left(\nabla \cdot\left(\mathbf{u}_{c}+\delta \hat{\mathbf{u}}\left(\mathbf{u}_{c}\right)\right), w_{c}\right)+\left(a \delta \hat{p}\left(\mathbf{u}_{c}\right), w_{c}\right) \\
& =\left(b^{*}-a \delta \bar{p}-\nabla \cdot \delta \overline{\mathbf{u}}, w_{c}\right) \quad \forall w_{c} \in W_{c}, \\
& \left(d^{-1}\left(\mathbf{u}_{c}+\delta \hat{\mathbf{u}}\left(\mathbf{u}_{c}\right)\right), \mathbf{v}_{c}+\delta \hat{\mathbf{u}}\left(\mathbf{v}_{c}\right)\right) \\
& +\left(a \delta \hat{p}\left(\mathbf{u}_{c}\right), \delta \hat{p}\left(\mathbf{v}_{c}\right)\right)+\left(\alpha \mathbf{u}_{c} \cdot \nu, \mathbf{v}_{c} \cdot \nu\right)_{\Gamma_{R}} \\
& -\left(p_{c}, \nabla \cdot\left(\mathbf{v}_{c}+\delta \hat{\mathbf{u}}\left(\mathbf{v}_{c}\right)\right)\right)-\left(a p_{c}, \delta \hat{p}\left(\mathbf{v}_{c}\right)\right) \\
& =\left(c^{*}-d^{-1} \delta \overline{\mathbf{u}}, \mathbf{v}_{c}\right)+\left(\delta \bar{p}, \nabla \cdot \mathbf{v}_{c}\right)-\left(g_{R}, \mathbf{v}_{c} \cdot \nu\right)_{\Gamma_{R}} \quad \forall \mathbf{v}_{c} \in \mathbf{V}_{c} .
\end{aligned}
$$

The final solution is given then by

$$
\begin{aligned}
& p=p_{c}+\delta \tilde{p}\left(p_{c}\right)+\delta \hat{p}\left(\mathbf{u}_{c}\right)+\delta \bar{p} \\
& \mathbf{u}=\mathbf{u}_{c}+\delta \tilde{\mathbf{u}}\left(p_{c}\right)+\delta \hat{\mathbf{u}}\left(\mathbf{u}_{c}\right)+\delta \overline{\mathbf{u}}+\mathbf{v}_{g_{N}} .
\end{aligned}
$$

It remains to show that indeed (4.10)-(4.11) or, equivalently, (4.12)-(4.13) has a unique solution from which to construct the solution $p$ and $\mathbf{u}$.

THEOREM 4.6. There exists a unique solution to (4.10)-(4.11) or, equivalently, to (4.12)-(4.13). Moreover, there is some constant $C$, independent of the coarse mesh $\mathcal{T}_{H}$ and the specific decomposition of $W \times \mathbf{V}$ selected, such that

$$
\left\|p_{c}\right\|_{0}+\left\|\mathbf{u}_{c}\right\|_{H(\text { div })} \leq C\left\{\|b\|_{0}+\|c\|_{0}+\left\|\mathbf{v}_{g_{N}}\right\|_{H(\text { div })}+\left\|g_{R}\right\|_{1 / 2, \partial \Omega}\right\} .
$$

Proof. Rather than trying to show the inf-sup condition for the system (4.12)(4.13) with its bilinear form $\left(\nabla \cdot\left(\mathbf{v}_{c}+\delta \hat{\mathbf{u}}\left(\mathbf{v}_{c}\right)\right), w_{c}\right)+\left(a \delta \hat{p}\left(\mathbf{v}_{c}\right), w_{c}\right)$, we use a more direct route. From Corollary 4.3 , we have $(p, \mathbf{u}) \in W \times\left(\mathbf{V}+\mathbf{v}_{g_{N}}\right)$ solving the original system. We uniquely decompose $p=p_{c}+\delta p \in W_{c} \oplus \delta W$ and $\mathbf{u}-\mathbf{v}_{g_{N}}=\mathbf{u}_{c}+\delta \mathbf{u} \in \mathbf{V}_{c} \oplus \delta \mathbf{V}$. By construction, $\left(p_{c}, \mathbf{u}_{c}\right) \in W_{c} \times \mathbf{V}_{c}$ is a solution to (4.12)-(4.13).

To demonstrate the uniqueness of the solution, consider the difference of two solutions, which is equivalent to setting all constant terms to zero and showing that there is only the trivial solution. Take the test functions $w_{c}=p_{c}$ and $\mathbf{v}_{c}=\mathbf{u}_{c}$ and sum the equations to conclude that $\mathbf{u}_{c}+\delta \hat{\mathbf{u}}\left(\mathbf{u}_{c}\right)=\delta \tilde{\mathbf{u}}\left(p_{c}\right)=0$ and, by the uniqueness of the decomposition $\mathbf{V}_{c} \oplus \delta \mathbf{V}$, that $\mathbf{u}_{c}=0$ and thus also $\delta \hat{p}\left(\mathbf{u}_{c}\right)=0$. Since $\nabla \cdot \mathbf{V}=W$, equations (4.7) and (4.11) imply that $p_{c}+\delta \tilde{p}\left(p_{c}\right)=0$, and thus $p_{c}=0$ and uniqueness is established.

We use (3.3) and (3.10) to bound

$$
\left\|p_{c}\right\|_{0} \leq C\|p\|_{0} \quad \text { and } \quad\left\|\mathbf{u}_{c}\right\|_{H(\operatorname{div})} \leq C\|\mathbf{u}\|_{H(\operatorname{div})} .
$$

Finally, Corollary 4.3 bounds these terms as required. 
5. Numerical approximation. In the previous section, we demonstrated that the $\delta$-problems (4.4)-(4.9) and the upscaled problem (4.12)-(4.13) are well-posed, uniformly with respect to $\mathcal{T}_{H}$. In this section, we construct an efficient computational algorithm, exploiting the structure exposed in the previous two sections. Namely, we exploit that the $\delta$-problems are local and thus easily solved computationally and that the global upscaled problem on $\mathcal{T}_{H}$ is relatively small compared to the full fine-scale problem (2.6)-(2.7) itself.

We present a class of discretizations based on standard mixed spaces. Our class of discretizations includes the particularly pertinent low order discretization described in $[1,2]$ and later in section 7 .

We now consider $\mathcal{T}_{H}$ as a coarse mesh. For approximation purposes, we assume that it is chosen of conforming simplexes, rectangular parallelepipeds, or prisms such that, for simplicity, $\bar{\Gamma}_{N}$ is the union of coarse edges or faces. Let

$$
H=\max _{E_{c} \in \mathcal{T}_{H}} \operatorname{diam}\left(E_{c}\right) .
$$

On each $E_{c} \in \mathcal{T}_{H}$, let $\mathcal{T}_{h}\left(E_{c}\right)$ be a fine mesh sufficient to resolve the coefficients of the problem, and define

$$
h=\max _{E_{c} \in \mathcal{T}_{H}} \max _{\delta E \in \mathcal{T}_{h}\left(E_{c}\right)} \operatorname{diam}(\delta E) .
$$

Then $\mathcal{T}_{H, h}=\cup_{E_{c} \in \mathcal{T}_{H}} \mathcal{T}_{h}\left(E_{c}\right)$ is the full fine mesh. The meshes need not match across boundaries of coarse elements.

5.1. Two-scale conforming approximation spaces. From among any of the usual mixed finite element spaces for second order elliptic equations, such as those of $[28,24,12,10,11,14,13]$, we select the coarse space $W_{H}^{*} \times \mathbf{V}_{H}^{*} \subset W \times \mathbf{V}$ on the mesh $\mathcal{T}_{H}$, with $\mathbf{V}_{H}^{*}$ satisfying the homogeneous Neumann boundary condition on $\Gamma_{N}$. In all the usual spaces,

$$
\nabla \cdot \mathbf{V}_{H}^{*}=W_{H}^{*}
$$

and piecewise discontinuous constants $W_{c}^{1} \subset W_{H}^{*}$.

On each coarse element $E_{c} \in \mathcal{T}_{H}$, we similarly select from among any of the usual mixed finite element spaces the $\delta$-space $\delta W_{h}\left(E_{c}\right) \times\left.\delta \mathbf{V}_{h}\left(E_{c}\right) \subset(W \times \mathbf{V})\right|_{E_{c}}$ on the mesh $\mathcal{T}_{h}\left(E_{c}\right)$, with $\delta W_{h}\left(E_{c}\right) \perp 1$ and $\delta \mathbf{V}_{h}\left(E_{c}\right)$ satisfying the homogeneous Neumann boundary condition on $\partial E_{c}$. Merging these spaces results in $\delta W_{h} \times \delta \mathbf{V}_{h}$ over the entire domain $\Omega$. Then $\delta W_{h} \perp W_{c}^{1}$ and

$$
\nabla \cdot \delta \mathbf{V}_{h}=\delta W_{h} .
$$

For simplicity, we take the same mixed space for each coarse element, although this assumption could be relaxed.

The overall two-scale mixed spaces are then defined to be

$$
W_{H, h}=W_{H}^{*}+\delta W_{h} \quad \text { and } \quad \mathbf{V}_{H, h}=\mathbf{V}_{H}^{*}+\delta \mathbf{V}_{h} .
$$

However, it is possible for general combinations of mixed spaces that the coarse and $\delta$-spaces are not linearly independent. The following construction suffices to rectify the problem. First, complete a basis for $\delta W_{h} \cap W_{H}^{*}$ to a basis for $W_{H}^{*}$ and then define $W_{H}$ as the span of the extra vectors. Similarly, we complete a basis for $\delta \mathbf{V}_{h} \cap \mathbf{V}_{H}^{*}$ to a basis for $\mathbf{V}_{H}^{*}$ and use the extra vectors to define $\mathbf{V}_{H}$. 
To summarize our construction, our two-scale finite element spaces are and satisfy

$$
\begin{aligned}
& W_{H, h}=W_{H}^{*}+\delta W_{h}=W_{H} \oplus \delta W_{h} \subset W, \\
& \mathbf{V}_{H, h}=\mathbf{V}_{H}^{*}+\delta \mathbf{V}_{h}=\mathbf{V}_{H} \oplus \delta \mathbf{V}_{h} \subset \mathbf{V},
\end{aligned}
$$

where

$$
\nabla \cdot \delta \mathbf{V}_{h}=\delta W_{h} \quad \text { and } \quad \nabla \cdot \mathbf{V}_{H, h}=W_{H, h}
$$

Our spaces are conforming in the sense that both $W_{H}$ and $\delta W_{h}$ are subspaces of $W$, and $\mathbf{V}_{H}$ and $\delta \mathbf{V}_{h}$ are subspaces of $\mathbf{V}$ and $\delta \mathbf{V}^{1}$, respectively, and thus have the required $H(\operatorname{div})$ smoothness and satisfy the requisite boundary conditions.

However, it is not necessarily the case that $W_{H} \subset W_{c}$ and $\delta W_{h} \subset \delta W$ nor that $\mathbf{V}_{H} \subset \mathbf{V}_{c}$ and $\delta \mathbf{V}_{h} \subset \delta \mathbf{V}$. In section 3.1, we made a few arbitrary choices. We could, for example, have chosen $W_{H}=W_{c}$ and then defined $\delta W$ in such a way that both $\delta W_{h} \subset \delta W$ and (3.3) hold, perhaps after assuming the restriction on the grid mentioned in section 6 . We might similarly be able to decompose $\mathbf{V}^{s}$ in such a way that $\mathbf{V}_{H} \subset \mathbf{V}_{c}$ and $\delta \mathbf{V}_{h} \subset \delta \mathbf{V}$. Then the mixed spaces would be fully conforming in the two-scale sense. However, there appears to be no advantage to such a construction, so we will not attempt it here.

5.2. The discrete equations in computable form. The key to efficient implementation is to determine the $\delta$-operators' actions only on the finite element basis for $\mathbf{V}_{H}$. We call such solutions numerical Green's functions, since they give the response of the system to a "unit" disturbance, which on the numerical level is a coarse-scale basis function.

Let $\left\{w_{H, i}\right\}_{i}$ and $\left\{\mathbf{v}_{H, j}\right\}_{j}$ be finite element bases for $W_{H}$ and $\mathbf{V}_{H}$, respectively. One property of a finite element basis is that the support of any basis function is relatively small. Expand

$$
p_{H}=\sum_{i} p_{i} w_{H, i} \quad \text { and } \quad \mathbf{u}_{H}=\sum_{j} u_{j} \mathbf{v}_{H, j} .
$$

Then to compute, for example,

$$
\delta \hat{\mathbf{u}}\left(\mathbf{u}_{H}\right)=\sum_{j} u_{j} \delta \hat{\mathbf{u}}\left(\mathbf{v}_{H, j}\right)
$$

requires only the numerical Green's functions $\delta \hat{\mathbf{u}}\left(\mathbf{v}_{H, j}\right)$ for each $j$.

The numerical scheme has three main steps. The first step is to compute the solutions to the following problems.

Constant part of the approximate $\delta$-solution operator. Find $\delta \bar{p}_{h} \in \delta W_{h}$ and $\delta \overline{\mathbf{u}}_{h} \in$ $\delta \mathbf{V}_{h}$ such that

$$
\begin{array}{ll}
\left(a \delta \bar{p}_{h}, \delta w_{h}\right)+\left(\nabla \cdot \delta \overline{\mathbf{u}}_{h}, \delta w_{h}\right)=\left(b^{*}, \delta w_{h}\right) & \forall \delta w_{h} \in \delta W_{h}, \\
\left(d^{-1} \delta \overline{\mathbf{u}}_{h}, \delta \mathbf{v}_{h}\right)-\left(\delta \bar{p}_{h}, \nabla \cdot \delta \mathbf{v}_{h}\right)=\left(c^{*}, \delta \mathbf{v}_{h}\right) & \forall \delta \mathbf{v}_{h} \in \delta \mathbf{V}_{h} .
\end{array}
$$

$W_{H}$-linear part of the approximate $\delta$-solution operator. For $w_{H, i}$ in a basis for $W_{H}$, find $\delta \tilde{p}_{h, i} \in \delta W_{h}$ and $\delta \tilde{\mathbf{u}}_{h, i} \in \delta \mathbf{V}_{h}$ such that

$$
\begin{array}{ll}
\left(a\left(w_{H, i}+\delta \tilde{p}_{h, i}\right), \delta w_{h}\right)+\left(\nabla \cdot \delta \tilde{\mathbf{u}}_{h, i}, \delta w_{h}\right)=0 & \forall \delta w_{h} \in \delta W_{h}, \\
\left(d^{-1} \delta \tilde{\mathbf{u}}_{h, i}, \delta \mathbf{v}_{h}\right)-\left(w_{H, i}+\delta \tilde{p}_{h, i}, \nabla \cdot \delta \mathbf{v}_{h}\right)=0 & \forall \delta \mathbf{v}_{h} \in \delta \mathbf{V}_{h} .
\end{array}
$$


$\mathbf{V}_{H}$-linear part of the approximate $\delta$-solution operator. For $\mathbf{v}_{H, j}$ in a basis for $\mathbf{V}_{H}$, find $\delta \hat{p}_{h, j} \in \delta W_{h}$ and $\delta \hat{\mathbf{u}}_{h, j} \in \delta \mathbf{V}_{h}$ such that

$$
\begin{array}{ll}
\left(a \delta \hat{p}_{h, j}, \delta w_{h}\right)+\left(\nabla \cdot\left(\mathbf{v}_{H, j}+\delta \hat{\mathbf{u}}_{h, j}\right), \delta w_{h}\right)=0 & \forall \delta w_{h} \in \delta W_{h} \\
\left(d^{-1}\left(\mathbf{v}_{H, j}+\delta \hat{\mathbf{u}}_{h, j}\right), \delta \mathbf{v}_{h}\right)-\left(\delta \hat{p}_{h, j}, \nabla \cdot \delta \mathbf{v}_{h}\right)=0 & \forall \delta \mathbf{v}_{h} \in \delta \mathbf{V}_{h} .
\end{array}
$$

These problems are quick and efficient to solve, since they are relatively quite small due to their local nature. That is, we actually solve them on each coarse element independently. For example, we know that for the standard mixed spaces, $\mathbf{v}_{H, j}$ is supported on at most two coarse elements, $E_{c}^{1}$ and $E_{c}^{2}$. Thus, to evaluate $\delta \hat{\mathbf{u}}\left(\mathbf{v}_{H, j}\right)$, we solve (5.6)-(5.7) twice, with all spaces and integrals restricted to $E_{c}^{k}$ for $k=1,2$. Then in fact $\delta \hat{\mathbf{u}}\left(\mathbf{v}_{H, j}\right)$ is the combination of the two solutions $\left.\delta \hat{\mathbf{u}}\right|_{E_{c}^{k}}\left(\left.\mathbf{v}_{H, j}\right|_{E_{c}^{k}}\right)$ on $E_{c}^{k}, k=1,2$. On each coarse element, each linear system in (5.2)-(5.7) has the same matrix, and only the so-called right-hand side vector varies. Thus it is reasonable to use a direct solver for these problems. Moreover, they parallelize trivially. Since these are square linear systems, existence and uniqueness of a solution follow from uniqueness, which follow in the usual way from the fact that $\nabla \cdot \delta \mathbf{V}_{h}=\delta W_{h}$.

Then we have the implicit expressions

$$
\begin{aligned}
& \delta p_{h}=\delta \tilde{p}_{h}\left(p_{H}\right)+\delta \hat{p}_{h}\left(\mathbf{u}_{H}\right)+\delta \bar{p}_{h}=\sum_{i} p_{i} \delta \tilde{p}_{h, i}+\sum_{j} u_{j} \delta \hat{p}_{h, j}+\delta \bar{p}_{h}, \\
& \delta \mathbf{u}_{h}=\delta \tilde{\mathbf{u}}_{h}\left(p_{H}\right)+\delta \hat{\mathbf{u}}_{h}\left(\mathbf{u}_{H}\right)+\delta \overline{\mathbf{u}}_{h}=\sum_{i} p_{i} \delta \tilde{\mathbf{u}}_{h, i}+\sum_{j} u_{j} \delta \hat{\mathbf{u}}_{h, j}+\delta \overline{\mathbf{u}}_{h},
\end{aligned}
$$

since at this stage of the computation $p_{i}$ and $u_{j}$ are not known.

The second main step is to compute the solution to the upscaled equation. We approximate (4.12)-(4.13) in the symmetric case by restricting to the finite element basis: Find $p_{H} \in W_{H}$ and $\mathbf{u}_{H} \in \mathbf{V}_{H}$ such that

$$
\begin{array}{lll}
\left(a\left(p_{H}+\delta \tilde{p}\left(p_{H}\right)\right), w_{H}+\delta \tilde{p}\left(w_{H}\right)\right)+\left(d^{-1} \delta \tilde{\mathbf{u}}\left(p_{H}\right), \delta \tilde{\mathbf{u}}\left(w_{H}\right)\right) & \\
\quad & +\left(\nabla \cdot\left(\mathbf{u}_{H}+\delta \hat{\mathbf{u}}\left(\mathbf{u}_{H}\right)\right), w_{H}\right)+\left(a \delta \hat{p}\left(\mathbf{u}_{H}\right), w_{H}\right) & \\
= & \left(b^{*}-a \delta \bar{p}_{h}-\nabla \cdot \delta \overline{\mathbf{u}}_{h}, w_{H}\right) & \\
\left(d^{-1}\left(\mathbf{u}_{H}+\delta \hat{\mathbf{u}}\left(\mathbf{u}_{H}\right)\right), \mathbf{v}_{H}+\delta \hat{\mathbf{u}}\left(\mathbf{v}_{H}\right)\right) & \\
\quad+\left(a \delta \hat{p}\left(\mathbf{u}_{H}\right), \delta \hat{p}\left(\mathbf{v}_{H}\right)\right)+\left(\alpha \mathbf{u}_{H} \cdot \nu, \mathbf{v}_{H} \cdot \nu\right)_{\Gamma_{R}} & \\
\quad & -\left(p_{H}, \nabla \cdot\left(\mathbf{v}_{H}+\delta \hat{\mathbf{u}}\left(\mathbf{v}_{H}\right)\right)\right)-\left(a p_{H}, \delta \hat{p}\left(\mathbf{v}_{H}\right)\right) & \\
= & \left(c^{*}-d^{-1} \delta \overline{\mathbf{u}}_{h}, \mathbf{v}_{H}\right)-\left(\delta \bar{p}_{h}, \nabla \cdot \mathbf{v}_{H}\right)-\left(g_{R}, \mathbf{v}_{H} \cdot \nu\right)_{\Gamma_{R}} & \forall \mathbf{v}_{H} \in \mathbf{V}_{H} .
\end{array}
$$

By following the computations in section 4.3 , we easily see that a similar finite element approximation of the asymmetric formulation (4.10)-(4.11) is equivalent to (5.10)(5.11). Either problem is the same size as a full finite element approximation of (2.6)-(2.7) over the coarse space $W_{H} \times \mathbf{V}_{H}$.

The final main step is to construct the solution using (5.8)-(5.9):

$$
\begin{aligned}
& p_{h}=p_{H}+\delta p_{h} \in W_{H, h}, \\
& \mathbf{u}_{h}=\mathbf{u}_{H}+\delta \mathbf{u}_{h}+\mathbf{v}_{g_{N}} \in \mathbf{V}_{H, h}+\mathbf{v}_{g_{N}} .
\end{aligned}
$$

5.3. An equivalent form for the discrete equations. It should be noted that our procedure is an efficient implementation of the algebraically equivalent mixed 
finite element method corresponding to (2.6)-(2.7), which is to find $\mathbf{u}_{h} \in \mathbf{V}_{H, h}+\mathbf{v}_{g_{N}}$ and $p_{h} \in W_{H, h}$ such that

$$
\begin{array}{ll}
\left(a p_{h}, w_{h}\right)+\left(\nabla \cdot \mathbf{u}_{h}, w_{h}\right)=\left(b, w_{h}\right) & \forall w_{h} \in W_{H, h}, \\
\left(d^{-1} \mathbf{u}_{h}, \mathbf{v}_{h}\right)+\left(\alpha \mathbf{u}_{h} \cdot \nu, \mathbf{v}_{h} \cdot \nu\right)_{\Gamma_{R}}-\left(p_{h}, \nabla \cdot \mathbf{v}_{h}\right) & \\
\quad=\left(c, \mathbf{v}_{h}\right)-\left(g_{R}, \mathbf{v}_{h} \cdot \nu\right)_{\Gamma_{R}} & \forall \mathbf{v}_{h} \in \mathbf{V}_{H, h} .
\end{array}
$$

Existence and uniqueness of a solution follow from uniqueness, which follows from the linear independence of $W_{H} \times \mathbf{V}_{H}$ and $\delta W_{h} \times \delta \mathbf{V}_{h}$ and the fact that $\nabla \cdot \mathbf{V}_{H, h}=W_{H, h}$.

From (5.14)-(5.15), we can conclude existence and uniqueness of (5.10)-(5.11), since $p_{h}=p_{H}+\delta p_{h}$ and $\mathbf{u}_{h}=\mathbf{u}_{H}+\delta \mathbf{u}_{h}$ give $p_{H}$ and $\mathbf{u}_{H}$, which satisfy the system.

6. Analysis of the approximation error. We begin this section with some notation. For $M$, a subspace of $L^{2}$, we denote by $\mathcal{P}_{M}: L^{2} \rightarrow M$ the orthogonal $L^{2}$-projection, based on the decomposition $L^{2}=M \oplus M^{\perp}$. We contrast this with $\tilde{\mathcal{P}}_{M}: L^{2} \rightarrow M$ from Proposition 3.1, which was based on a possibly nonorthogonal decomposition $L^{2}=M \oplus N$.

At this point, we require some uniformity of the discrete decomposition. Let $\tilde{\mathcal{P}}_{W_{H}}: W_{H, h} \rightarrow W_{H}$ and $\tilde{\mathcal{P}}_{\delta W_{h}}: W_{H, h} \rightarrow \delta W_{h}$ be the projections associated with the decomposition $W_{H, h}=W_{H} \oplus \delta W_{h}$. We assume that there is a constant $C$ such that

$$
\left\|\tilde{\mathcal{P}}_{\delta W_{h}}\right\| \leq C \text {. }
$$

Note that then also

$$
\left\|\tilde{\mathcal{P}}_{W_{H}}\right\|=\left\|I-\tilde{\mathcal{P}}_{\delta W_{h}}\right\| \leq 1+C .
$$

It is not difficult to ensure (6.1). The simplest possibility is that $W_{H} \perp \delta W_{h}$ so that $\tilde{\mathcal{P}}_{\delta W_{h}}=\mathcal{P}_{\delta W_{h}}$ and we can take $C=1$. This holds for certain choices of mixed spaces but not for others. Another possibility is to enforce uniformity on the two-scale mesh $\mathcal{T}_{H, h}$. Suppose that as $H \rightarrow 0$, we insist that $H / h$ remains fixed. If we also assume that the coarse and fine element shapes remain fixed, then it is clear by a scaling argument that (6.1) will hold on each coarse element and thus globally. Moreover, we can even allow the element shapes to change as long as they do not change too badly, such as being the images of a reference element under a uniformly bounded family of affine maps with uniformly bounded inverses.

Let $K \geq 1$ and $L \geq 1$ denote the approximation orders of the coarse spaces $\mathbf{V}_{H}^{*}$ and $W_{H}^{*}$, respectively. That is, for some constant $C$ and for any $\mathbf{v} \in \mathbf{V}$ and $w \in W$,

$$
\begin{gathered}
\inf _{\mathbf{v}_{H} \in \mathbf{V}_{H}^{*}}\left\|\mathbf{v}-\mathbf{v}_{H}\right\|_{0} \leq C\|\mathbf{v}\|_{m} H^{m}, \quad 0 \leq m \leq K, \\
\inf _{w_{H} \in W_{H}^{*}}\left\|w-w_{H}\right\|_{0} \leq C\|w\|_{i} H^{i}, \quad 0 \leq i \leq L .
\end{gathered}
$$

For all the usual mixed spaces, $L=K$ or $L=K-1$. It is also true that

$$
\inf _{\mathbf{v}_{H} \in \mathbf{V}_{H}^{*}}\left\|\left(\mathbf{v}-\mathbf{v}_{H}\right) \cdot \nu\right\|_{0, \Gamma_{R}} \leq C\|\mathbf{v} \cdot \nu\|_{m, \Gamma_{R}} H^{m}, \quad 0 \leq m \leq K .
$$

Similarly, let $k \geq 1$ and $\ell \geq 1$ denote the approximation orders of the $\delta$-spaces $\delta \mathbf{V}_{h}$ and $\delta W_{h}$, respectively.

Lemma 6.1. Given any $w \in W$,

$$
\left\|w-\mathcal{P}_{W_{H, h}} w\right\|_{0} \leq C\|w\|_{i+j} H^{i} h^{j}, \quad 0 \leq i \leq \max (0, L-j), 0 \leq j \leq \ell .
$$


Proof. For each $E_{c} \in \mathcal{T}_{H}$, we note that in all the usual mixed spaces $W_{H}^{*}$ restricted to $E_{c}$ consists of polynomials of full degree at least $L-1$ that are discontinuous across $\partial E_{c}$. Thus from standard polynomial approximation results, we compute

$$
\begin{aligned}
\left\|w-\mathcal{P}_{W_{H, h}} w\right\|_{0, E_{c}} & =\inf _{w_{H} \in W_{H}^{*}} \inf _{\delta w_{h} \in \delta W_{h}}\left\|w-w_{H}-\delta w_{h}\right\|_{0, E_{c}} \\
& \leq C \inf _{w_{H} \in W_{H}^{*}}\left\|w-w_{H}\right\|_{j, E_{c}} h^{j} \\
& \leq C\|w\|_{i+j, E_{c}} H^{i} h^{j}
\end{aligned}
$$

wherein $0 \leq j \leq \ell$ and then $0 \leq i \leq \max (0, L-j)$.

Approximation in $\mathbf{V}_{H, h}$ is more delicate, as we need to preserve divergence properties.

6.1. A mixed method $\boldsymbol{\pi}$-operator. All the usual mixed spaces $\check{W}_{\eta} \times \check{\mathbf{V}}_{\eta}$ have projection operators $\check{\pi}: \mathbf{V} \cap H^{1}(\Omega) \rightarrow \check{\mathbf{V}}_{\eta}$ such that

$$
\begin{aligned}
& \nabla \cdot \check{\pi} \mathbf{v}=\mathcal{P}_{\check{W}_{\eta}} \nabla \cdot \mathbf{v}, \\
& \|\mathbf{v}-\check{\pi} \mathbf{v}\| \leq C\|\mathbf{v}\|_{i} \eta^{i}, \quad 1 \leq i \leq m,
\end{aligned}
$$

where $C$ is a constant independent of the mesh spacing $\eta$ and $m$ is the approximation order of the space $\check{\mathbf{V}}_{\eta}$. Moreover, on $\partial \Omega$,

$$
\check{\pi} \mathbf{v} \cdot \nu=\mathcal{P}_{\check{\mathbf{V}}_{\eta} \cdot \nu} \mathbf{v} \cdot \nu .
$$

We have the associated operators

$$
\begin{aligned}
& \pi_{H}: \mathbf{V} \cap H^{1}(\Omega) \rightarrow \mathbf{V}_{H}^{*}, \\
& \delta \pi_{E_{c}, h}: \delta \mathbf{V}\left(E_{c}\right) \cap H^{1}\left(E_{c}\right) \rightarrow \delta \mathbf{V}_{h}\left(E_{c}\right) \quad \forall E_{c} \in \mathcal{T}_{H},
\end{aligned}
$$

and also $\delta \pi_{h}$, defined by combining the $\delta \pi_{E_{c}, h}$. Then for any $\mathbf{v} \in \mathbf{V}$ and $\delta \mathbf{v} \in \delta \mathbf{V}^{1}$,

$$
\begin{aligned}
& \nabla \cdot \pi_{H} \mathbf{v}=\mathcal{P}_{W_{H}^{*}} \nabla \cdot \mathbf{v}, \\
& \nabla \cdot \delta \pi_{h} \delta \mathbf{v}=\mathcal{P}_{\delta W_{h}} \nabla \cdot \delta \mathbf{v} .
\end{aligned}
$$

Our goal now is to define a similar operator for the two-scale space $W_{H, h} \times \mathbf{V}_{H, h}$. Let $\mathbf{v} \in \mathbf{V} \cap H^{1}(\Omega)$. On each $E_{c} \in \mathcal{T}_{H}$, let

$$
\delta w=\tilde{\mathcal{P}}_{\delta W_{h}} \mathcal{P}_{W_{H, h}} \nabla \cdot \mathbf{v} .
$$

Define $\delta \mathbf{v}^{p}=\nabla \varphi$, where $\varphi \in H^{1}\left(E_{c}\right)$ satisfies (3.4)-(3.5) with the given $\delta w$. Then, because of (3.1), the Poincaré inequality constant is independent of $H$ and $h$, so elliptic regularity [19] gives us the bound

$$
\|\varphi\|_{2, E_{c}} \leq C\left\|\tilde{\mathcal{P}}_{\delta W_{h}} \mathcal{P}_{W_{H, h}} \nabla \cdot \mathbf{v}\right\|_{0, E_{c}}
$$

where a simple scaling argument shows that $C$ depends on the shape of $E_{c}$ but not on its size. Thus also

$$
\left\|\delta \mathbf{v}^{p}\right\|_{1, E_{c}} \leq C\left\|\tilde{\mathcal{P}}_{\delta W_{h}} \mathcal{P}_{W_{H, h}} \nabla \cdot \mathbf{v}\right\|_{0, E_{c}}
$$

and we conclude that we can apply $\delta \pi_{h}$ to $\delta \mathbf{v}^{p}$.

Definition 6.2. Let $\pi: \mathbf{V} \cap H^{1}(\Omega) \rightarrow \mathbf{V}_{H, h}=\mathbf{V}_{H}^{*}+\delta \mathbf{V}_{h}$ be defined by

$$
\pi \mathbf{v}=\pi_{H}\left(\mathbf{v}-\delta \mathbf{v}^{p}\right)+\delta \pi_{h} \delta \mathbf{v}^{p},
$$

where $\delta \mathbf{v}^{p}=\nabla \varphi$ and $\varphi$ satisfies (3.4)-(3.5) with $\delta w=\tilde{\mathcal{P}}_{\delta W_{h}} \mathcal{P}_{W_{H, h}} \nabla \cdot \mathbf{v}$.

This operator, while well defined, is not a projection. 
Proposition 6.3. It follows that

$$
\begin{array}{ll}
\nabla \cdot \pi \mathbf{v}=\mathcal{P}_{W_{H, h}} \nabla \cdot \mathbf{v} & \text { on } \Omega, \\
\pi \mathbf{v} \cdot \nu=\pi_{H} \mathbf{v} \cdot \nu & \text { on } \partial \Omega .
\end{array}
$$

Proof. Since $\mathcal{P}_{W_{H, h}}=\left(\tilde{\mathcal{P}}_{W_{H}}+\tilde{\mathcal{P}}_{\delta W_{h}}\right) \mathcal{P}_{W_{H, h}}$ and $\mathcal{P}_{W_{H}^{*}} \mathcal{P}_{W_{H, h}}=\mathcal{P}_{W_{H}^{*}}$, we compute

$$
\begin{aligned}
\nabla \cdot \pi \mathbf{v} & =\nabla \cdot \pi_{H}\left(\mathbf{v}-\delta \mathbf{v}^{p}\right)+\nabla \cdot \delta \pi_{h} \delta \mathbf{v}^{p} \\
& =\mathcal{P}_{W_{H}^{*}} \nabla \cdot\left(\mathbf{v}-\delta \mathbf{v}^{p}\right)+\mathcal{P}_{\delta W_{h}} \nabla \cdot \delta \mathbf{v}^{p} \\
& =\mathcal{P}_{W_{H}^{*}} \nabla \cdot \mathbf{v}-\mathcal{P}_{W_{H}^{*}} \tilde{\mathcal{P}}_{\delta W_{h}} \mathcal{P}_{W_{H, h}} \nabla \cdot \mathbf{v}+\mathcal{P}_{\delta W_{h}} \tilde{\mathcal{P}}_{\delta W_{h}} \mathcal{P}_{W_{H, h}} \nabla \cdot \mathbf{v} \\
& =\mathcal{P}_{W_{H}^{*}} \nabla \cdot \mathbf{v}-\mathcal{P}_{W_{H}^{*}}\left(I-\tilde{\mathcal{P}}_{W_{H}}\right) \mathcal{P}_{W_{H, h}} \nabla \cdot \mathbf{v}+\tilde{\mathcal{P}}_{\delta W_{h}} \mathcal{P}_{W_{H, h}} \nabla \cdot \mathbf{v} \\
& =\left(\tilde{\mathcal{P}}_{W_{H}}+\tilde{\mathcal{P}}_{\delta W_{h}}\right) \mathcal{P}_{W_{H, h}} \nabla \cdot \mathbf{v} \\
& =\mathcal{P}_{W_{H, h}} \nabla \cdot \mathbf{v} .
\end{aligned}
$$

By (6.7), we see that

$$
\pi \mathbf{v} \cdot \nu=\pi_{H}\left(\mathbf{v}-\delta \mathbf{v}^{p}\right) \cdot \nu+\delta \pi_{h} \delta \mathbf{v}^{p} \cdot \nu=\pi_{H} \mathbf{v} \cdot \nu,
$$

since $\delta \mathbf{v}^{p} \cdot \nu=0$ on $\partial \Omega$.

LEMMA 6.4. If (3.1) and (6.1) hold, then for $\mathbf{v} \in \mathbf{V} \cap H^{1}(\Omega)$,

$$
\begin{aligned}
& \|\mathbf{v}-\pi \mathbf{v}\|_{0} \leq C\|\mathbf{v}\|_{m} H^{m}, \quad 1 \leq m \leq K, \\
& \|(\mathbf{v}-\pi \mathbf{v}) \cdot \nu\|_{0, \Gamma_{R}} \leq C\|\mathbf{v} \cdot \nu\|_{m, \Gamma_{R}} H^{m}, \quad 0 \leq m \leq K .
\end{aligned}
$$

Proof. We construct $\delta \mathbf{v}^{p} \in \delta \mathbf{V}^{p}$ as in the definition of $\pi$. Then for $1 \leq m \leq K$,

$$
\begin{aligned}
\|\mathbf{v}-\pi \mathbf{v}\|_{0} & =\left\|\mathbf{v}-\pi_{H} \mathbf{v}+\pi_{H} \delta \mathbf{v}^{p}-\delta \pi_{h} \delta \mathbf{v}^{p}\right\|_{0} \\
& \leq\left\|\mathbf{v}-\pi_{H} \mathbf{v}\right\|_{0}+\left\|\pi_{H} \delta \mathbf{v}^{p}-\delta \mathbf{v}^{p}\right\|_{0}+\left\|\delta \mathbf{v}^{p}-\delta \pi_{h} \delta \mathbf{v}^{p}\right\|_{0} \\
& \leq C\left\{\|\mathbf{v}\|_{m} H^{m}+\sum_{E_{c} \in \mathcal{T}_{H}}\left(\left\|\delta \mathbf{v}^{p}\right\|_{1, E_{c}} H+\left\|\delta \mathbf{v}^{p}\right\|_{1, E_{c}} h\right)\right\} \\
& \leq C\left\{\|\mathbf{v}\|_{m} H^{m}+\left\|\tilde{\mathcal{P}}_{\delta W_{h}} \mathcal{P}_{W_{H, h}} \nabla \cdot \mathbf{v}\right\|_{0} H\right\},
\end{aligned}
$$

by (6.8). The Bramble-Hilbert lemma [15, 7] implies that for any $0 \leq i \leq L$,

$$
\left\|\tilde{\mathcal{P}}_{\delta W_{h}} \mathcal{P}_{W_{H, h}} \nabla \cdot \mathbf{v}\right\|_{0}=\left\|\left(I-\tilde{\mathcal{P}}_{W_{H}}\right) \mathcal{P}_{W_{H, h}} \nabla \cdot \mathbf{v}\right\|_{0} \leq C\|\nabla \cdot \mathbf{v}\|_{i} H^{i},
$$

since the operator $\left(I-\tilde{\mathcal{P}}_{W_{H}}\right) \mathcal{P}_{W_{H, h}}$ is uniformly bounded by (6.1), and it preserves polynomials of the appropriate degree. With $i=m-1, i$ is in the range $0 \leq i \leq L$ ( $L=K$ or $L=K-1)$, and we obtain the required first estimate.

The second estimate follows from Proposition 6.3 and its approximation properties, (6.7) and (6.5).

6.2. Error analysis. The equation for the error is given by (2.6)-(2.7) with test functions in $W_{H, h} \times \mathbf{V}_{H, h}$ minus (5.14)-(5.15), which is

$$
\begin{array}{lll}
(6.9) & \left(a\left(p-p_{h}\right), w\right)+\left(\nabla \cdot\left(\mathbf{u}-\mathbf{u}_{h}\right), w\right)=0 & \forall w \in W_{H, h} \\
(6.10) & \left(d^{-1}\left(\mathbf{u}-\mathbf{u}_{h}\right), \mathbf{v}\right)+\left(\alpha\left(\mathbf{u}-\mathbf{u}_{h}\right) \cdot \nu, \mathbf{v} \cdot \nu\right)_{\Gamma_{R}}=\left(p-p_{h}, \nabla \cdot \mathbf{v}\right) & \forall \mathbf{v} \in \mathbf{V}_{H, h}
\end{array}
$$


Theorem 6.5. If (3.1) and (6.1) hold, and $a \in W^{1, \infty}(\Omega)$, then the two-scale approximation satisfies the error bounds

$$
\begin{aligned}
& \left\|\sqrt{a}\left(\mathcal{P}_{W_{H, h}} p-p_{h}\right)\right\|_{0}+\left\|\mathbf{u}-\mathbf{u}_{h}\right\|_{0}+\left\|\sqrt{\alpha}\left(\mathbf{u}-\mathbf{u}_{h}\right) \cdot \nu\right\|_{0, \Gamma_{R}} \\
& \quad \leq C\left\{\|p\|_{i+j} H^{i} h^{j+1}+\left(\left\|\mathbf{u}-\mathbf{v}_{g_{N}}\right\|_{m}+\left\|\left(\mathbf{u}-\mathbf{v}_{g_{N}}\right) \cdot \nu\right\|_{m, \Gamma_{R}}\right) H^{m}\right\}, \\
& \left\|\mathcal{P}_{W_{H, h}} p-p_{h}\right\|_{0} \leq C\left\{\left\|\mathbf{u}-\mathbf{u}_{h}\right\|_{0}+\left\|\sqrt{\alpha}\left(\mathbf{u}-\mathbf{u}_{h}\right) \cdot \nu\right\|_{0, \Gamma_{R}}\right\} \\
& \left\|p-p_{h}\right\|_{0} \leq C\left\{\left\|\mathcal{P}_{W_{H, h}} p-p_{h}\right\|_{0}+\|p\|_{i+j} H^{i} h^{j}\right\} \\
& \left\|\nabla \cdot\left(\mathbf{u}-\mathbf{u}_{h}\right)\right\|_{0} \leq C\left\{\left\|\sqrt{a}\left(\mathcal{P}_{W_{H, h}} p-p_{h}\right)\right\|_{0}+\|p\|_{i+j} H^{i} h^{j+1}+\|\nabla \cdot \mathbf{u}\|_{i+j} H^{i} h^{j}\right\},
\end{aligned}
$$

wherein $0 \leq i \leq \max (0, L-j), 0 \leq j \leq \ell$, and $1 \leq m \leq K$. Moreover, if $\alpha=0$ or $\Gamma_{R}=\emptyset$, and if $h$ is sufficiently small, then

$$
\left\|\mathcal{P}_{W_{H, h}} p-p_{h}\right\|_{0} \leq C\left\{\left\|\nabla \cdot\left(\mathbf{u}-\mathbf{u}_{h}\right)\right\|_{0} h+\|p\|_{i+j} H^{i} h^{j+1}+\left\|\mathbf{u}-\mathbf{u}_{h}\right\|_{0} H\right\} .
$$

Note that these are optimal order estimates, since $L=K$ or $L=K-1$. Moreover, $\left\|\mathcal{P}_{W_{H, h}} p-p_{h}\right\|_{0}$ is superconvergent if $\alpha=0$ or $\Gamma_{R}=\emptyset$ and $h$ is sufficiently small.

Proof. For notational convenience, let us define

$$
\pi \mathbf{u} \equiv \pi\left(\mathbf{u}-\mathbf{v}_{g_{N}}\right)+\mathbf{v}_{g_{N}} .
$$

The sum of the equations (6.9)-(6.10) with

$$
w=\mathcal{P}_{W_{H, h}} p-p_{h} \in W_{H, h} \quad \text { and } \quad \mathbf{v}=\pi \mathbf{u}-\mathbf{u}_{h} \in \mathbf{V}_{H, h},
$$

because of Proposition 6.3 and the fact that $\nabla \cdot \mathbf{V}_{H, h}=W_{H, h}$, results in

$$
\begin{aligned}
&(a(\left.\left.\mathcal{P}_{W_{H, h}} p-p_{h}\right), \mathcal{P}_{W_{H, h}} p-p_{h}\right)+\left(d^{-1}\left(\mathbf{u}-\mathbf{u}_{h}\right), \mathbf{u}-\mathbf{u}_{h}\right) \\
& \quad+\left(\alpha\left(\mathbf{u}-\mathbf{u}_{h}\right) \cdot \nu,\left(\mathbf{u}-\mathbf{u}_{h}\right) \cdot \nu\right)_{\Gamma_{R}} \\
& \quad=\left(a\left(\mathcal{P}_{W_{H, h}} p-p\right), \mathcal{P}_{W_{H, h}} p-p_{h}\right)+\left(d^{-1}\left(\mathbf{u}-\mathbf{u}_{h}\right), \mathbf{u}-\pi \mathbf{u}\right) \\
& \quad+\left(\alpha\left(\mathbf{u}-\mathbf{u}_{h}\right) \cdot \nu,(\mathbf{u}-\pi \mathbf{u}) \cdot \nu\right)_{\Gamma_{R}} .
\end{aligned}
$$

If $\bar{a} \in W_{H, h}$ is the piecewise discontinuous constant average of $a$ over the fine mesh $\mathcal{T}_{H, h}$, then

$$
\begin{aligned}
& \left(a\left(\mathcal{P}_{W_{H, h}} p-p\right), \mathcal{P}_{W_{H, h}} p-p_{h}\right)=\left((a-\bar{a})\left(\mathcal{P}_{W_{H, h}} p-p\right), \mathcal{P}_{W_{H, h}} p-p_{h}\right) \\
& \quad \leq C\|a\|_{W^{1, \infty}(\Omega)} h\left\|\mathcal{P}_{W_{H, h}} p-p\right\|_{0}\left\|\mathcal{P}_{W_{H, h}} p-p_{h}\right\|_{0} .
\end{aligned}
$$

Thus for any $\epsilon>0$,

$$
\begin{aligned}
& \left\|\sqrt{a}\left(\mathcal{P}_{W_{H, h}} p-p_{h}\right)\right\|_{0}+\left\|\mathbf{u}-\mathbf{u}_{h}\right\|_{0}+\left\|\sqrt{\alpha}\left(\mathbf{u}-\mathbf{u}_{h}\right) \cdot \nu\right\|_{0, \Gamma_{R}} \\
& \quad \leq C_{\epsilon}\left\{\left\|p-\mathcal{P}_{W_{H, h}} p\right\|_{0} h+\|\mathbf{u}-\pi \mathbf{u}\|_{0}+\|(\mathbf{u}-\pi \mathbf{u}) \cdot \nu\|_{0, \Gamma_{R}}\right\}+\epsilon\left\|\mathcal{P}_{W_{H, h}} p-p_{h}\right\|_{0} .
\end{aligned}
$$

Standard elliptic lift arguments can be used to estimate $\mathcal{P}_{W_{H, h}} p-p_{h}$. That is, we solve (3.6)-(3.8) for $\psi$ with $w_{c}$ replaced by $\mathcal{P}_{W_{H, h}} p-p_{h}$ and take $\mathbf{v}=\pi \nabla \psi$. Then $\nabla \cdot \mathbf{v}=\mathcal{P}_{W_{H, h}} p-p_{h}$ and

$$
\begin{aligned}
& \|\mathbf{v}\|_{0} \leq\|\nabla \psi\|_{0}+\|\nabla \psi-\pi \nabla \psi\|_{0} \leq C\|\psi\|_{2} \leq C\left\|\mathcal{P}_{W_{H, h}} p-p_{h}\right\|_{0}, \\
& \|\mathbf{v} \cdot \nu\|_{0, \Gamma_{R}} \leq\|\nabla \psi \cdot \nu\|_{0, \Gamma_{R}}+\left\|\left(\nabla \psi-\pi_{H} \nabla \psi\right) \cdot \nu\right\|_{0, \Gamma_{R}} \\
& \quad \leq C\|\psi\|_{2} \leq C\left\|\mathcal{P}_{W_{H, h}} p-p_{h}\right\|_{0},
\end{aligned}
$$


using (6.7). Then (6.10) implies that

$$
\begin{aligned}
\left\|\mathcal{P}_{W_{H, h}} p-p_{h}\right\|_{0}^{2} & =\left(d^{-1}\left(\mathbf{u}-\mathbf{u}_{h}\right), \mathbf{v}\right)+\left(\alpha\left(\mathbf{u}-\mathbf{u}_{h}\right) \cdot \nu, \mathbf{v} \cdot \nu\right)_{\Gamma_{R}} \\
& \leq C\left\{\left\|\mathbf{u}-\mathbf{u}_{h}\right\|_{0}\|\mathbf{v}\|_{0}+\left\|\sqrt{\alpha}\left(\mathbf{u}-\mathbf{u}_{h}\right) \cdot \nu\right\|_{0, \Gamma_{R}}\|\mathbf{v} \cdot \nu\|_{0, \Gamma_{R}}\right. \\
& \leq C\left\{\left\|\mathbf{u}-\mathbf{u}_{h}\right\|_{0}+\left\|\sqrt{\alpha}\left(\mathbf{u}-\mathbf{u}_{h}\right) \cdot \nu\right\|_{0, \Gamma_{R}}\right\}\left\|\mathcal{P}_{W_{H, h}} p-p_{h}\right\|_{0}
\end{aligned}
$$

and, with Lemmas 6.1 and 6.4, the first three estimates of the theorem follow.

If $\alpha=0$ or $\Gamma_{R}=\emptyset$, we replace (3.6)-(3.8) by

$$
\begin{array}{ll}
a \psi-\nabla \cdot d \nabla \psi=\mathcal{P}_{W_{H, h}} p-p_{h} & \text { in } \Omega, \\
-d \nabla \psi \cdot \nu=0 & \text { on } \Gamma_{N}, \\
\psi=0 & \text { on } \Gamma_{R},
\end{array}
$$

and we modify the argument as follows [16]:

$$
\begin{aligned}
\left\|\mathcal{P}_{W_{H, h}} p-p_{h}\right\|_{0}^{2} & =\left(\mathcal{P}_{W_{H, h}} p-p_{h}, a \psi-\nabla \cdot d \nabla \psi\right) \\
& =\left(a\left(\mathcal{P}_{W_{H, h}} p-p_{h}\right), \psi\right)-\left(\mathcal{P}_{W_{H, h}} p-p_{h}, \nabla \cdot \pi d \nabla \psi\right),
\end{aligned}
$$

and, using (6.10),

$$
\begin{aligned}
\left(\mathcal{P}_{W_{H, h}} p-p_{h}, \nabla \cdot \pi d \nabla \psi\right) & =\left(d^{-1}\left(\mathbf{u}-\mathbf{u}_{h}\right), \pi d \nabla \psi\right) \\
& =\left(\mathbf{u}-\mathbf{u}_{h}, \nabla \psi\right)-\left(d^{-1}\left(\mathbf{u}-\mathbf{u}_{h}\right), d \nabla \psi-\pi d \nabla \psi\right) \\
& =-\left(\nabla \cdot\left(\mathbf{u}-\mathbf{u}_{h}\right), \psi\right)-\left(d^{-1}\left(\mathbf{u}-\mathbf{u}_{h}\right), d \nabla \psi-\pi d \nabla \psi\right)
\end{aligned}
$$

and, for $w \in W_{H, h}$ arbitrary, by (6.9),

$$
\begin{aligned}
\left(\nabla \cdot\left(\mathbf{u}-\mathbf{u}_{h}\right), \psi\right)= & \left(\nabla \cdot\left(\mathbf{u}-\mathbf{u}_{h}\right), \psi-w\right)-\left(a\left(\mathcal{P}_{W_{H, h}} p-p_{h}\right), w\right) \\
& -\left(a\left(p-\mathcal{P}_{W_{H, h}} p\right), w\right) \\
= & \left(\nabla \cdot\left(\mathbf{u}-\mathbf{u}_{h}\right), \psi-w\right)-\left(a\left(\mathcal{P}_{W_{H, h}} p-p_{h}\right), \psi\right) \\
& -\left(a\left(\mathcal{P}_{W_{H, h}} p-p_{h}\right), w-\psi\right)-\left((a-\bar{a})\left(p-\mathcal{P}_{W_{H, h}} p\right), w\right) .
\end{aligned}
$$

Since a good choice of $w$ implies

$$
\|w-\psi\|_{0} \leq C\|\psi\|_{1} h \quad \text { and } \quad\|w\|_{0} \leq C\|\psi\|_{1},
$$

we have that

$$
\begin{aligned}
\left\|\mathcal{P}_{W_{H, h}} p-p_{h}\right\|_{0}^{2} \leq & C\left\{\left(\left\|\nabla \cdot\left(\mathbf{u}-\mathbf{u}_{h}\right)\right\|_{0}+\left\|\mathcal{P}_{W_{H, h}} p-p_{h}\right\|_{0}+\left\|p-\mathcal{P}_{W_{H, h}} p\right\|_{0}\right)\|\psi\|_{1} h\right. \\
& \left.+\left\|\mathbf{u}-\mathbf{u}_{h}\right\|_{0}\|\psi\|_{2} H\right\} \\
\leq & C\left\{\left(\left\|\nabla \cdot\left(\mathbf{u}-\mathbf{u}_{h}\right)\right\|_{0}+\left\|\mathcal{P}_{W_{H, h}} p-p_{h}\right\|_{0}+\left\|p-\mathcal{P}_{W_{H, h}} p\right\|_{0}\right) h\right. \\
& \left.+\left\|\mathbf{u}-\mathbf{u}_{h}\right\|_{0} H\right\}\left\|\mathcal{P}_{W_{H, h}} p-p_{h}\right\|_{0}
\end{aligned}
$$

and the final result of the theorem follows for $h$ sufficiently small.

Finally, (6.9) with $w=\nabla \cdot\left(\pi \mathbf{u}-\mathbf{u}_{h}\right) \in W_{H, h}$ implies that

$$
\begin{aligned}
\left\|\nabla \cdot\left(\pi \mathbf{u}-\mathbf{u}_{h}\right)\right\|_{0}^{2}= & -\left(a\left(\mathcal{P}_{W_{H, h}} p-p_{h}\right), \nabla \cdot\left(\pi \mathbf{u}-\mathbf{u}_{h}\right)\right) \\
& -\left((a-\bar{a})\left(p-\mathcal{P}_{W_{H, h}} p\right), \nabla \cdot\left(\pi \mathbf{u}-\mathbf{u}_{h}\right)\right) .
\end{aligned}
$$

Since $\nabla \cdot(\mathbf{u}-\pi \mathbf{u})=\left(I-\mathcal{P}_{W_{H, h}}\right) \nabla \cdot \mathbf{u}$, which approximates as in (6.6), the divergence estimate of the theorem follows. 
In the special case $a=\alpha=0$, we obtain optimality of the finite element approximation $\mathbf{u}_{h}$ to $\mathbf{u}$ in the energy norm subject to the appropriate divergence constraint.

TheOREM 6.6. If $a=\alpha=0$ and (3.1) and (6.1) hold, then

$$
\begin{aligned}
& \left\|d^{-1 / 2}\left(\mathbf{u}-\mathbf{u}_{h}\right)\right\|_{0} \leq \inf _{\nabla \cdot \mathbf{v}_{h}=\mathcal{P}_{W_{H, h} \in \mathbf{v}_{H, h}} \nabla \cdot\left(\mathbf{u}-\mathbf{v}_{g_{N}}\right)}\left\|d^{-1 / 2}\left(\mathbf{u}-\mathbf{v}_{g_{N}}-\mathbf{v}_{h}\right)\right\|_{0} \leq C\left\|\mathbf{u}-\mathbf{v}_{g_{N}}\right\|_{m} H^{m}, \\
& \nabla \cdot \mathbf{u}_{h}=\mathcal{P}_{W_{H, h}} \nabla \cdot \mathbf{u}, \\
& \left\|\mathcal{P}_{W_{H, h}} p-p_{h}\right\|_{0} \leq C\left\|\mathbf{u}-\mathbf{u}_{h}\right\|_{0}, \\
& \left\|\mathcal{P}_{W_{H, h}} p-p_{h}\right\|_{0} \leq C\left\{\left\|\nabla \cdot\left(\mathbf{u}-\mathbf{u}_{h}\right)\right\|_{0} h+\left\|\mathbf{u}-\mathbf{u}_{h}\right\|_{0} H\right\} \\
& \left\|p-p_{h}\right\|_{0} \leq C\left\{\left\|\mathcal{P}_{W_{H, h}} p-p_{h}\right\|_{0}+\|p\|_{i+j} H^{i} h^{j}\right\}
\end{aligned}
$$

wherein $0 \leq i \leq \max (0, L-j), 0 \leq j \leq \ell$, and $1 \leq m \leq K$.

Proof. For any $\mathbf{v}_{h} \in \mathbf{V}_{H, h}$ such that $\nabla \cdot \mathbf{v}_{h}=\mathcal{P}_{W_{H, h}} \nabla \cdot\left(\mathbf{u}-\mathbf{v}_{g_{N}}\right)$, take

$$
\begin{aligned}
& w=\mathcal{P}_{W_{H, h}} p-p_{h} \in W_{H, h}, \\
& \mathbf{v}=\left(\mathbf{u}-\mathbf{u}_{h}\right)-\left(\mathbf{u}-\mathbf{v}_{g_{N}}-\mathbf{v}_{h}\right) \in \mathbf{V}_{H, h}
\end{aligned}
$$

in (6.9)-(6.10). Then with $a=\alpha=0$, the sum of the equations implies that

$$
\begin{aligned}
\left(d^{-1}\left(\mathbf{u}-\mathbf{u}_{h}\right), \mathbf{u}-\mathbf{u}_{h}\right) & =\left(d^{-1}\left(\mathbf{u}-\mathbf{u}_{h}\right), \mathbf{u}-\mathbf{v}_{g_{N}}-\mathbf{v}_{h}\right) \\
& \leq\left\|d^{-1 / 2}\left(\mathbf{u}-\mathbf{u}_{h}\right)\right\|_{0}\left\|d^{-1 / 2}\left(\mathbf{u}-\mathbf{v}_{g_{N}}-\mathbf{v}_{h}\right)\right\|_{0}
\end{aligned}
$$

so that

$$
\left\|d^{-1 / 2}\left(\mathbf{u}-\mathbf{u}_{h}\right)\right\|_{0} \leq \inf _{\mathbf{v}_{h}}\left\|d^{-1 / 2}\left(\mathbf{u}-\mathbf{v}_{g_{N}}-\mathbf{v}_{h}\right)\right\|_{0} \leq C\left\|\mathbf{u}-\mathbf{v}_{g_{N}}-\pi\left(\mathbf{u}-\mathbf{v}_{g_{N}}\right)\right\|_{0}
$$

since $\pi\left(\mathbf{u}-\mathbf{v}_{g_{N}}\right)$ satisfies the divergence constraint by Proposition 6.3. Lemma 6.4 gives the required approximation result for the first estimate of the theorem.

Now $a=0$ and (6.9) imply that $\nabla \cdot \mathbf{u}_{h}=\mathcal{P}_{W_{H, h}} \nabla \cdot \mathbf{u}$, giving the second result of the theorem. The final estimates follow as in the previous proof.

The underlying assumption in the error analysis, and the tacit assumption in all similar subgrid methods mentioned in the introduction, is that the finest grid, scale $h$, resolves the fine-scale details of the solution. We see this here in the Sobolev norms appearing in the error estimates. If $h$ does not resolve the subgrid problems, then we cannot expect a good approximation.

Under this tacit assumption, we proved two main results. First, in Theorem 6.6, the solution is optimally approximated in the finite element space subject to the finescale divergence constraint. Thus the approximation is no worse than using only a coarse-scale approximation (up to questions of the scale of the divergence constraint). It is presumably much better, as numerical results show $[1,2,4]$. Second, the pressure is approximated on the finest scale, up to a higher order coarse $H$ error term. This is a strict improvement over merely solving on the coarse scale. Moreover, these improvements are achieved for negligible additional numerical cost compared to the coarse-scale solution and much less cost than the fine-scale solution itself $[1,4]$. 
The error estimates of this subsection appear to explain the numerical results that have appeared elsewhere: that they are amazingly accurate for reasonable problems where the fine grid resolves the coefficients of the problem $[1,2,4]$. The error estimates also explain why numerical results break down in certain difficult cases in which $h$ does not resolve the problem well. If $h$ and $H$ are relatively large compared to the Sobolev norms of the solution, the method is not expected to work well. This appears to be the main factor limiting the utility of the method in practice. As an example, in [2], a numerical test was presented involving Darcy flow in a porous medium with a long thin high permeability streak. This is a very difficult problem to resolve numerically on a coarse grid. The streak introduces channelized flow, which means that the derivatives of the solution are large, and so by Theorem 6.5 or 6.6 , we neither expect nor see good results. (It should be noted, however, that the upscaling technique of this paper is not entirely unsuccessful in resolving the solution of even this very difficult problem.)

7. Two special cases. A particularly pertinent choice of spaces was studied numerically in $[1,2]$. On the coarse mesh, we approximate $\left(p_{c}, \mathbf{u}_{c}\right)$ in the two-dimensional BDM-1 or three-dimensional BDDF-1 mixed finite element spaces $[12,10]$. The space of scalars $W_{H}^{*}$ consists of piecewise discontinuous constants, and the space of vectors $\mathbf{V}_{H}^{*}$ is second order accurate and has linear fluxes on the edges or faces of coarse elements.

On each coarse element $E_{c} \in \mathcal{T}_{H}$, we approximate $(\delta \bar{p}, \delta \overline{\mathbf{u}}),(\delta \tilde{p}, \delta \tilde{\mathbf{u}})$, and $(\delta \hat{p}, \delta \hat{\mathbf{u}})$ in the RT-0 spaces [28]. These approximate with piecewise discontinuous constants for $\delta W_{h}\left(E_{c}\right)$ and with constant fluxes on each interior edge or face for $\delta \mathbf{V}_{h}\left(E_{c}\right)$.

In this case, $\mathbf{V}_{H}^{*} \cap \delta \mathbf{V}_{h}=\{0\}$, so $\mathbf{V}_{H}=\mathbf{V}_{H}^{*}$ requires no attention. Also, $W_{H, h}=$ $W_{h}^{*}$, the space of piecewise discontinuous constants over the fine mesh $\mathcal{T}_{H, h}$. We note that $W_{H}^{*} \perp \delta W_{h}$, so $W_{H}=W_{H}^{*}$ and condition (6.1) holds. However, it is not so simple to compute with $\delta W_{h}\left(E_{c}\right)$, since such functions are supported in all of $E_{c}$. Fortunately, a careful implementation of the scheme [2] allows one to avoid working over $\delta W_{h}\left(E_{c}\right)$ and instead work over the full space $\delta W_{h}\left(E_{c}\right)+\operatorname{span}\{1\}$ of piecewise discontinuous constants over $\mathcal{T}_{h}\left(E_{c}\right)$. Now $K=2$ and $L=k=\ell=1$, so Theorem 6.5 takes the following simple form.

THEOREM 7.1. If $a \in W^{1, \infty}(\Omega)$ and (3.1) holds, the BDDF-1(BDM-1)/RT-0 two-scale approximation satisfies the error bounds

$$
\begin{aligned}
& \left\|\sqrt{a}\left(\mathcal{P}_{W_{H, h}} p-p_{h}\right)\right\|_{0}+\left\|\mathbf{u}-\mathbf{u}_{h}\right\|_{0}+\left\|\sqrt{\alpha}\left(\mathbf{u}-\mathbf{u}_{h}\right) \cdot \nu\right\|_{0, \Gamma_{R}} \\
& \quad \leq C\left\{\|p\|_{1} h^{2}+\left(\left\|\mathbf{u}-\mathbf{v}_{g_{N}}\right\|_{2}+\left\|\left(\mathbf{u}-\mathbf{v}_{g_{N}}\right) \cdot \nu\right\|_{2, \Gamma_{R}}\right) H^{2}\right\} \leq C H^{2}, \\
& \left\|\mathcal{P}_{W_{H, h}} p-p_{h}\right\|_{0} \leq C\left\{\left\|\mathbf{u}-\mathbf{u}_{h}\right\|_{0}+\left\|\sqrt{\alpha}\left(\mathbf{u}-\mathbf{u}_{h}\right) \cdot \nu\right\|_{0, \Gamma_{R}}\right\} \leq C H^{2}, \\
& \left\|p-p_{h}\right\|_{0} \leq C\left\{\left\|\mathcal{P}_{W_{H, h}} p-p_{h}\right\|_{0}+\|p\|_{1} h\right\} \leq C\left(H^{2}+h\right), \\
& \left\|\nabla \cdot\left(\mathbf{u}-\mathbf{u}_{h}\right)\right\|_{0} \leq C\left\{\left\|\sqrt{a}\left(\mathcal{P}_{W_{H, h}} p-p_{h}\right)\right\|_{0}+\|p\|_{1} h^{2}+\|\nabla \cdot \mathbf{u}\|_{1} h\right\} \leq C\left(H^{2}+h\right) .
\end{aligned}
$$

Moreover, if $\alpha=0$ or $\Gamma_{R}=\emptyset$, and $h$ is sufficiently small, then

$$
\left\|\mathcal{P}_{W_{H, h}} p-p_{h}\right\|_{0} \leq C\left\{\left\|\nabla \cdot\left(\mathbf{u}-\mathbf{u}_{h}\right)\right\|_{0} h+\|p\|_{1} h^{2}+\left\|\mathbf{u}-\mathbf{u}_{h}\right\|_{0} H\right\} \leq C\left(H^{3}+h^{2}\right) .
$$

If $H^{2} \sim h$ as $H \rightarrow 0$, then $p$ and $\mathbf{u}$ are resolved on the fine scale to order $h$. Hence relatively good numerical approximation results have been obtained $[1,3,4,2]$.

A second special choice is to use RT-0 spaces on both scales. In this case, $K=$ $L=k=\ell=1$, and Theorem 6.5 would suggest that $p$ and $\mathbf{u}$ are approximated only on the coarse scale to order $H$. Although we retain the optimality of the solution 
in the energy norm under the appropriate conditions, the numerical approximation results are not nearly as good as in the previous special case (see [5]).

\section{REFERENCES}

[1] T. Arbogast, Numerical subgrid upscaling of two-phase flow in porous media, in Numerical Treatment of Multiphase Flows in Porous Media, Lecture Notes in Phys. 552, Z. Chen, R. E. Ewing, and Z.-C. Shi, eds., Springer, Berlin, 2000, pp. 35-49.

[2] T. Arbogast, Implementation of a locally conservative numerical subgrid upscaling scheme for two-phase Darcy flow, Comput. Geosci., 6 (2002), pp. 453-481.

[3] T. Arbogast and S. Bryant, Efficient forward modeling for DNAPL site evaluation and remediation, in Computational Methods in Water Resources XIII, L. R. Bentley, J. F. Sykes, C. A. Brebbia, W. G. Gray, and G. F. Pinder, eds., Balkema, Rotterdam, 2000, pp. 161-166.

[4] T. Arbogast and S. L. Bryant, A two-scale numerical subgrid technique for waterflood simulations, SPE J., 7 (2002), pp. 446-457.

[5] T. Arbogast, S. E. Minkoff, And P. T. Keenan, An operator-based approach to upscaling the pressure equation, in Computational Methods in Water Resources XII, Vol. 1, V. N. Burganos, G. P. Karatzas, A. C. Payatakes, C. A. Brebbia, W. G. Gray, and G. F. Pinder, eds., Computational Mechanics Publications, Southampton, UK, 1998, pp. 405-412.

[6] I. BABUŠKA, The finite element method with Lagrangian multipliers, Numer. Math., 20 (1973), pp. 179-192.

[7] S. C. Brenner And L. R. Scott, The Mathematical Theory of Finite Element Methods, Springer-Verlag, New York, 1994.

[8] F. BREzzI, On the existence, uniqueness and approximation of saddle-point problems arising from Lagrangian multipliers, Rev. Française Automat. Informat. Recherche Opérationnelle Sér. Rouge, 8 (1974), pp. 129-151.

[9] F. BREzzI, Interacting with the subgrid world, in Proceedings of the Conference on Numerical Analysis, Dundee, Scotland, 1999.

[10] F. Brezzi, J. Douglas, Jr., R. Duràn, and M. Fortin, Mixed finite elements for second order elliptic problems in three variables, Numer. Math., 51 (1987), pp. 237-250.

[11] F. Brezzi, J. Douglas, JR., M. Fortin, and L. D. Marini, Efficient rectangular mixed finite elements in two and three space variables, RAIRO Modél. Math. Anal. Numér., 21 (1987), pp. 581-604.

[12] F. Brezzi, J. Douglas, Jr., And L. D. Marini, Two families of mixed elements for second order elliptic problems, Numer. Math., 47 (1985), pp. 217-235.

[13] F. Brezzi And M. Fortin, Mixed and Hybrid Finite Element Methods, Springer-Verlag, New York, 1991.

[14] Z. Chen and J. Douglas, JR., Prismatic mixed finite elements for second order elliptic problems, Calcolo, 26 (1989), pp. 135-148.

[15] P. Ciarlet, The Finite Element Method for Elliptic Problems, North-Holland, Amsterdam, 1978.

[16] J. Douglas, JR., And J. E. Roberts, Global estimates for mixed methods for second order elliptic equations, Math. Comp., 44 (1985), pp. 39-52.

[17] D. Gilbarg And N. S. Trudinger, Elliptic Partial Differential Equations of Second Order, Springer-Verlag, Berlin, 1983.

[18] V. Girault And J.-L. Lions, Two-grid finite-element schemes for the transient Navier-Stokes problem, M2AN Math. Model. Numer. Anal., 35 (2001), pp. 945-980.

[19] P. Grisvard, Elliptic Problems in Nonsmooth Domains, Pitman, Boston, 1985.

[20] T. Y. Hou AND X. H. Wu, A multiscale finite element method for elliptic problems in composite materials and porous media, J. Comput. Phys., 134 (1997), pp. 169-189.

[21] T. J. R. Hughes, Multiscale phenomena: Green's functions, the Dirichlet-to-Neumann formulation, subgrid scale models, bubbles and the origins of stabilized methods, Comput. Methods Appl. Mech. Engrg., 127 (1995), pp. 387-401.

[22] T. J. R. Hughes, G. R. Feisóo, L. Mazzei, And J.-B. Quincy, The variational multiscale method - A paradigm for computational mechanics, Comput. Methods Appl. Mech. Engrg., 166 (1998), pp. 3-24.

[23] T. J. R. Hughes, A. A. Oberai, and L. Mazzei, Large eddy simulation of turbulent channel flows by the variational multiscale method, Phys. Fluids, 13 (2001), pp. 1784-1799.

[24] J. C. NedÉLÉc, Mixed finite elements in $\mathbf{R}^{3}$, Numer. Math., 35 (1980), pp. 315-341.

[25] J. T. Oden And K. S. Vemaganti, Adaptive hierarchical modeling of heterogeneous structures, 
Phys. D, 133 (1999), pp. 404-415.

[26] J. T. OdEn AND K. S. Vemaganti, Estimation of local modeling error and goal-oriented adaptive modeling of heterogeneous materials. I. Error estimates and adaptive algorithms, J. Comput. Phys., 164 (2000), pp. 22-47.

[27] M. Peszynska, M. F. Wheeler, And I. Yotov, Mortar upscaling for multiphase flow in porous media, Comput. Geosci., 6 (2002), pp. 73-100.

[28] R. A. Raviart and J. M. Thomas, A mixed finite element method for 2 nd order elliptic problems, in Mathematical Aspects of Finite Element Methods, Lecture Notes in Math. 606, I. Galligani and E. Magenes, eds., Springer-Verlag, New York, 1977, pp. 292-315.

[29] W. Rudin, Functional Analysis, McGraw-Hill, New York, 1991.

[30] J. XU, Two-grid discretization techniques for linear and nonlinear PDEs, SIAM. J. Numer. Anal., 33 (1996), pp. 1759-1777.

[31] J. Xu, The method of subspace corrections, J. Comput. Appl. Math., 128 (2001), pp. 335-362. 